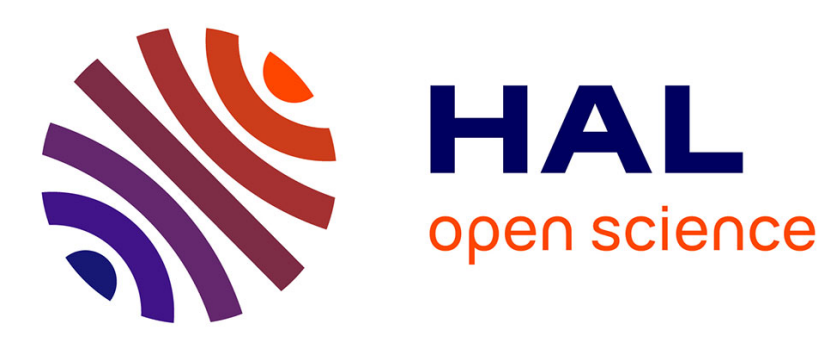

\title{
A forward-backward probabilistic algorithm for the incompressible Navier-Stokes equations
}

\author{
Antoine Lejay, Hernán Mardones González
}

\section{To cite this version:}

Antoine Lejay, Hernán Mardones González. A forward-backward probabilistic algorithm for the incompressible Navier-Stokes equations. Journal of Computational Physics, 2020, 420 (109689), 10.1016/j.jcp.2020.109689 . hal-02377108v3

\section{HAL Id: hal-02377108 \\ https://hal.inria.fr/hal-02377108v3}

Submitted on 15 Sep 2020

HAL is a multi-disciplinary open access archive for the deposit and dissemination of scientific research documents, whether they are published or not. The documents may come from teaching and research institutions in France or abroad, or from public or private research centers.
L'archive ouverte pluridisciplinaire $\mathbf{H A L}$, est destinée au dépôt et à la diffusion de documents scientifiques de niveau recherche, publiés ou non, émanant des établissements d'enseignement et de recherche français ou étrangers, des laboratoires publics ou privés. 


\title{
A forward-backward probabilistic algorithm for the incompressible Navier-Stokes equations
}

\author{
Antoine Lejay* Hernán Mardones González
}

May 21, 2020

\begin{abstract}
A novel probabilistic scheme for solving the incompressible Navier-Stokes equations is studied, in which we approximate a generalized nonlinear Feyman-Kac formula. The velocity field is interpreted as the mean value of a stochastic process ruled by Forward-Backward Stochastic Differential Equations (FBSDEs) driven by Brownian motion. Following an approach by Delbaen, Qiu and Tang introduced in 2015, the pressure term is obtained from the velocity by solving a Poisson problem as computing the expectation of an integral functional associated to an extra BSDE. The FBSDEs components are numerically solved by using a forward-backward algorithm based on Euler type schemes for the local time integration and the quantization of the increments of Brownian motion following the algorithm proposed by Delarue and Menozzi in 2006. Numerical results are reported on spatially periodic analytic solutions of the Navier-Stokes equations for incompressible fluids. We illustrate the proposed algorithm on a two dimensional Taylor-Green vortex and three dimensional Beltrami flows.
\end{abstract}

Keywords: Navier-Stokes equations $\bullet$ Brownian motion $\bullet$ Feynman-Kac formula $\bullet$ forwardbackward stochastic differential equations $\bullet$ incompressible fluids $\bullet$ numerical solution

\section{Introduction}

The Navier-Stokes equations system allows us to model the movement of fluids. It was introduced by C.-L. Navier in 1822 [52] and G. G. Stokes in 1849 [62] by incorporating a pressure term and the fluid viscosity to the Euler equations due to L.'Euler in 1757 [27]. Nowadays, technological and scientific computing developments provide a suitable way for the simulation of mathematical models, and in turn research and analysis of efficient numerical methodologies to approximate exact solutions. With this in mind, we study the numerical simulation of the Navier-Stokes equations for incompressible fluids in $\mathbb{R}^{d}$ (for space dimension $d \in\{2,3\}$ )

$$
\left\{\begin{array}{l}
\frac{\partial u}{\partial t}+(u \cdot \nabla) u=\nu \triangle u-\nabla p+f ; \quad 0<t \leq T \\
\nabla \cdot u=0, \quad u(0)=g
\end{array}\right.
$$

where $T>0$ is a fixed time, $\nu>0$ is the kinematic viscosity, $f$ is the external force field and $g$ is a given initial divergence-free vector field. The system of equations (1) describes the motion of an incompressible fluid by means of unknown fields of velocity $u(t, x) \in \mathbb{R}^{d}$ and pressure

*Université de Lorraine, CNRS, Inria, IECL, F-54000 Nancy, France; antoine.lejay@univ-lorraine.fr

$\dagger$ Universidad de La Serena, Facultad de Ciencias, Departamento de Matemáticas, La Serena, Chile; hmardo@ gmail.com 
$p(t, x) \in \mathbb{R}$ defined for each time $t \in[0, T]$ and position $x \in \mathbb{R}^{d}$. It involves a nonlinear convective term $(u \cdot \nabla) u$, a diffusion term $\nu \triangle u$ and the incompressibility condition $\nabla \cdot u=0$. Although being introduced in the nineteenth century, there are still open problems concerning the Navier-Stokes equations. Nonetheless it is usual to find works that deal with the fluid dynamics using the Navier-Stokes model (ocean modelling, atmosphere of Earth, gas dynamics, dynamic of storms, etc.) [28, 31, 9, 64. There exists a huge literature on computational fluid dynamics, and probabilistic numerical methods for nonlinear models have gained attention during the last decades.

The Itô stochastic differential equations (SDEs) introduced during the 1940s by K. Itô's seminal works [35, 36] are connected with partial differential equations (PDEs) by means of the celebrated Feynman-Kac formula due to R. Feynman [29] and M. Kac [38]. More recently, the theory of backward stochastic differential equations (BSDEs), initiated by É. Pardoux and S. Peng in [58], permits to obtain probabilistic representations for the solutions of a broad class of PDEs by means of systems of forward-backward SDEs (FBSDEs) through a nonlinear Feynman-Kac formula. Hence, the numerical solution of FBSDEs provides a stochastic algorithm to approximate solutions of nonlinear PDEs. Among the theory of SDEs, FBSDEs associated to the unsteady Navier-Stokes equations is a novel approach.

Let us assume that the solution $u$ of $(1)$ is known on a time interval $[0, T]$, which means that the pressure term $p$ is also known (the force field $f$ is considered as given). We define by $X$ the $d$-dimensional diffusion process associated to the differential operator $u \rightarrow(u \cdot \nabla) u+\nu \triangle u$. With $Y_{s}:=-u\left(T-s, X_{s}\right)$ and $Z_{s}:=-D u\left(T-s, X_{s}\right)$, the process $(X, Y, Z)$ is solution to the FBSDEs

$$
\left\{\begin{array}{l}
X_{s}=x+\int_{t}^{s} Y_{r} \mathrm{~d} r+\int_{t}^{s} \sqrt{2 \nu} \mathrm{d} W_{r}, \\
Y_{s}=-g\left(X_{T}\right)+\int_{s}^{T}\left[\nabla p\left(T-r, X_{r}\right)-f\left(T-r, X_{r}\right)\right] \mathrm{d} r-\int_{s}^{T} \sqrt{2 \nu} Z_{r} \mathrm{~d} W_{r},
\end{array}\right.
$$

where $W_{s}$ is a $d$-dimensional standard Brownian motion. The numerical solution of the incompressible Navier-Stokes equations (1) involves various difficulties due to the presence of a nonlinear term, the incompressibility condition as well as the computation of the pressure term. The simplified model obtained by removing the pressure term from the FBSDEs (2) is associated to the Burgers equation [8], which remains a nonlinear equation due to the term $(u \cdot \nabla) u$. Under appropriate conditions, the pressure term $p$ is given by solving a Poisson problem $-\triangle p=\mathcal{P}(u)$ for a given functional of the solution.

Recently F. Delbaen, J. Qiu and S. Tang introduced in [26] a new class of coupled FBSDEs associated to the incompressible Navier-Stokes equations. Since their probabilistic approach involves an extra BSDE defined on an infinite time interval for the stochastic representation of the pressure term, Delbaen et al. deduced an approximated solution to the velocity field by truncating the infinite interval of the associated FBSDEs system. Hence they proposed a numerical simulation algorithm, that follows a methodology of F. Delarue and S. Menozzi [24, 25] for FBSDEs, to simulate the incompressible Navier-Stokes equations in the whole Cartesian space.

Specialized literature on probabilistic representations to the solution of PDEs is presented through Feynman-Kac formulae or well by means of branching processes. In general, the deterministic solutions of particular equations are obtained through the expectation of functionals of stochastic processes. During the last years some probabilistic approaches have been proposed to deal with the Navier-Stokes equations. The random vortex methods study the incompressible Navier-Stokes system in vorticity form [15, 16, 47]. Branching processes and stochastic cascades present another way of interpretation [40, 4, 5, 53, 67. The stochastic Lagrangian paths provide additional representations [17, 37, 68]. Moreover, stochastic particle systems arise from the McKean-Vlasov equations in both two and three dimensional contexts [63, 33, 32]. Recently 
the systems of FBSDEs appear as a novel approach [22, 26]. The problem of relating boundary conditions on probabilistic representations presents additional challenges [6, 18, 50, 51].

The main motivation of this paper is to develop a suitable numerical framework formulated on the new probabilistic representation of Delbaen et al for solving the incompressible NavierStokes equation (1) in two and three spatial dimensions. The main point is to test if such a method is practically feasible, while Monte Carlo methods are in general difficult to implement for such non-linear equations. Monte Carlo methods however offer the advantage of being simple to interpret and to set-up. By considering the numerical solution associated to the system of FBSDEs and their approximation by mean values, we consider a backward regression based on the Euler-Maruyama scheme and the quantization method. The pressure gradient is recovered from the expected value of an integral functional of Brownian motion. To do this, we use the Riemann sum estimation and the quantization of the Brownian increments.

Finally, we test the performance of the novel stochastic algorithm to the simulation of spatially-periodic analytic solutions in the unsteady regime. In our simulations, the magnitude of Reynolds number range from 1 to 200. Our numerical tests show that the proposed scheme is practically implementable. However, it suffers from long run-times which motivate the development of ad hoc variance reduction schemes. This article is only a preliminary step to the development of a Monte Carlo method for solving the incompressible Navier-Stokes equation.

\section{Outline}

Section 2 details the link between FBSDEs driven by Brownian motion and deterministic PDEs through the nonlinear Feynman-Kac formula. The incompressible Navier-Stokes equations are associated to the novel FBSDEs system. Section 3 presents the numerical methodology for solving FBSDEs. We detail the approximation of mean values and the numerical treatment of integral functionals of Brownian motion. Section 4 illustrates the forward-backward probabilistic algorithm by solving a two-dimensional Taylor-Green vortex and three-dimensional Beltrami flows as numerical examples. Finally Section 5 is devoted to concluding remarks.

\section{Notations}

We use the following notations and conventions:

- We consider a filtered complete probability space $\left(\Omega, \mathcal{F},\left(\mathcal{F}_{t}\right)_{t \geq 0}, \mathbb{P}\right)$.

- For each $q \geq 1$, we set

$$
L_{\mathcal{F}}^{q}\left(\Omega ; \mathbb{R}^{d}\right):=\left\{\xi: \Omega \rightarrow \mathbb{R}^{d}: \xi \text { is } \mathcal{F} \text {-measurable and } \mathbb{E}\|\xi\|^{q}<\infty\right\} .
$$

- For each $m, n \in \mathbb{Z}_{+}$and $A \in \mathbb{R}^{m \times n}$, we denote by $A^{\top}$ its transpose matrix while $I_{m}$ represents the $m \times m$ identity matrix. The matrix $A \in \mathbb{R}^{n \times n}$ is said to be elliptic if there exists $\lambda>0$ such that

$$
\langle x, A x\rangle \geq \lambda\|x\| ; \quad \forall x \in \mathbb{R}^{n} .
$$

The set of $m \times m$ real valued symmetric non-negative matrices is

$$
\mathbb{S}_{+}^{m}:=\left\{Q \in \mathbb{R}^{m \times m}: Q=Q^{\top} \text { and }\langle x, Q x\rangle \geq 0 ; \quad \forall x \in \mathbb{R}^{m}\right\} .
$$

- The symbol " $\approx$ " means "is numerically approximated by".

- The symbol $\mathcal{O}$ means the order of estimation. For example, $h=\mathcal{O}\left(\delta^{2}\right)$ means that $h>0$ is depending on $\delta>0$ and less than $K \delta^{2}$, with constant $K$ independent of $\delta$. From now on $K>0$ stands for constants independent on the discretization parameters.

- The indicator function of a set $A$ is $\mathbf{1}_{A}$.

- In general, we use the Einstein summation convention through this paper. 
- The space $C^{k, \alpha}\left(\mathbb{R}^{d}, \mathbb{R}^{n}\right)$, or simply as $C^{k, \alpha}$, of $k$-continuously differentiable functions $\phi$ : $\mathbb{R}^{d} \rightarrow \mathbb{R}^{n}$ with $\alpha$ Hölder continuity for each $k$-order partial derivatives equipped with the norm

$$
\|\phi\|_{C^{k, \alpha}}:=\sup _{x \in \mathbb{R}^{d}}\|\phi(x)\|+\sum_{|\beta|=1}^{k} \sup _{x \in \mathbb{R}^{d}}\left\|D^{\beta} \phi(x)\right\|+\sum_{|\beta|=k} \sup _{x, y \in \mathbb{R}^{d}, x \neq y} \frac{\left\|D^{\beta} \phi(x)-D^{\beta} \phi(y)\right\|}{\|x-y\|^{\alpha}} .
$$

The multi-index notation is considered.

- For $T>0$ and $t \in[0, T)$ we denote by $C\left([t, T] ; \mathbb{R}^{m \times n}\right)$ the space of continuous functions $\psi$ with norm

$$
\|\psi\|_{C\left([t, T] ; \mathbb{R}^{m \times n}\right)}:=\sup _{s \in[t, T]}\|\psi(s)\| .
$$

Similarly we have the space $C\left([t, T] ; C^{k, \alpha}\right)$ of continuous functions with

$$
\|\psi\|_{C\left([t, T] ; C^{k, \alpha}\right)}:=\sup _{s \in[t, T]}\|\psi(s)\|_{C^{k, \alpha}} .
$$

- Let $L_{\mathcal{F}}^{2}\left(\Omega \times[t, T] ; \mathbb{R}^{m \times n}\right)$ the space of predictable stochastic processes $\Psi$ with values in $\mathbb{R}^{m \times n}$ suth that

$$
\mathbb{E}\left(\int_{t}^{T}\left\|\Psi_{s}\right\|^{2} d s\right)^{1 / 2}<\infty
$$

- Let $L_{\mathcal{F}}^{2}\left(\Omega ; C\left([t, T] ; \mathbb{R}^{m \times n}\right)\right)$ be the set of all $\left\{\mathcal{F}_{t}\right\}_{t \geq 0}$-progressively measurable stochastic processes $\Psi$ with continuous trajectories taking values in $\mathbb{R}^{m \times n}$ such that

$$
\mathbb{E} \sup _{s \in[t, T]}\left\|\Psi_{s}\right\|^{2}<\infty
$$

- For $m \in \mathbb{N}$ and $q \in[1, \infty)$ we denote by $L^{q}\left(\mathbb{R}^{\ell}\right)$ and $H^{m, q}\left(\mathbb{R}^{\ell}\right)$ the usual $\mathbb{R}^{\ell}$-valued Lebesgue and Sobolev spaces on $\mathbb{R}^{d}$, respectively. When $\ell=d$ we just write $L^{q}$ and $H^{m, q}$, or $H^{m}$ when $q=2$.

- The space $H_{\sigma}^{m}$ is the completion of $\left\{\phi \in C_{c}^{\infty}\left(\mathbb{R}^{d}\right): \operatorname{div} \phi=0\right\}$ under the norm

$$
\|\phi\|_{H_{\sigma}^{m}}:=\sup _{x \in \mathbb{R}^{d}}\|\phi(x)\|+\sum_{|\beta|=1}^{m} \sup _{x \in \mathbb{R}^{d}}\left\|D^{\beta} \phi(x)\right\| .
$$

The space $C\left([0, T] ; H_{\sigma}^{m}\right)$ of continuous functions $\psi$ is equipped with

$$
\|\psi\|_{C\left([0, T] ; H_{\sigma}^{m}\right)}:=\sup _{s \in[0, T]}\|\psi(s)\|_{H_{\sigma}^{m}} .
$$

\section{Forward-backward stochastic differential equations}

Diffusion processes are linked to PDEs through the celebrated Feynman-Kac formula. The theory of Itô SDEs together Backward SDEs constitute systems of FBSDEs that are connected with deterministic nonlinear PDEs through a nonlinear Feynman-Kac formula, generalizing the classical Feynman-Kac formula (see e.g. [1, 42, 10]).

Let $T>0, t \in[0, T)$ and $x \in \mathbb{R}^{d}$ be fixed. Consider the FBSDEs on $[t, T]$ of the form

$$
\left\{\begin{array}{l}
X_{s}=x+\int_{t}^{s} b\left(r, X_{r}, Y_{r}, Z_{r}\right) \mathrm{d} r+\int_{t}^{s} \sigma\left(r, X_{r}, Y_{r}\right) \mathrm{d} W_{r} \\
Y_{s}=g\left(X_{T}\right)+\int_{s}^{T} h\left(r, X_{r}, Y_{r}, Z_{r}\right) \mathrm{d} r-\int_{s}^{T} Z_{r} \mathrm{~d} W_{r}
\end{array}\right.
$$


where $b:[0, T] \times \mathbb{R}^{d} \times \mathbb{R}^{n} \times \mathbb{R}^{n \times m} \rightarrow \mathbb{R}^{d}, \sigma:[0, T] \times \mathbb{R}^{d} \times \mathbb{R}^{n} \rightarrow \mathbb{R}^{d \times m}, g: \mathbb{R}^{d} \rightarrow \mathbb{R}^{n}$, $h:[0, T] \times \mathbb{R}^{d} \times \mathbb{R}^{n} \times \mathbb{R}^{n \times m} \rightarrow \mathbb{R}^{n}$ are given and $W=\left(W^{1}, \ldots, W^{m}\right)^{\top}$ is a $m$-dimensional Wiener process defined on $\left(\Omega, \mathcal{F},\left(\mathcal{F}_{t}\right)_{t \geq 0}, \mathbb{P}\right)$. Here, $\mathcal{F}_{t \geq 0}$ is the natural filtration of the Wiener process $W$ augmented with $\mathbb{P}$-null sets. An adapted solution of the FBSDEs (3) is defined by a triple of processes

$$
(X, Y, Z) \in L_{\mathcal{F}}^{2}\left(\Omega ; C\left([t, T] ; \mathbb{R}^{d}\right)\right) \times L_{\mathcal{F}}^{2}\left(\Omega ; C\left([t, T] ; \mathbb{R}^{n}\right)\right) \times L_{\mathcal{F}}^{2}\left(\Omega \times[t, T] ; \mathbb{R}^{n \times m}\right)
$$

that satisfies $(3) \mathbb{P}$-almost surely. The BSDE in the system (3) depends on the diffusion process $X$ that solves the forward SDE. When the coefficients of the diffusion process $X$ do not depend on $Y$ nor $Z$, (3) is referred as a decoupled FBSDEs. Otherwise we have a coupled FBSDEs. From now on, we denote by $\left(X^{t, x}, Y^{t, x}, Z^{t, x}\right)$ to recall the dependence on the parameters $(t, x) \in$ $[0, T) \times \mathbb{R}^{d}$. We refer for example to $[57,23]$ for the classic theory of FBSDEs.

\subsection{Nonlinear Feynman-Kac formula}

As in the spirit of the Feynman-Kac formula, it is possible to obtain a probabilistic representation for the solution $u=\left(u_{1}, \ldots, u_{n}\right)^{\top}:[0, T] \times \mathbb{R}^{d} \rightarrow \mathbb{R}^{n}$ of the backward quasilinear PDE

$\left\{\begin{array}{l}\frac{\partial u}{\partial t}(t, x)+\mathcal{L}(t, x, u(t, x))+h(t, x, u(t, x), D u(t, x) \sigma(t, x, u(t, x)))=0 \quad ; \forall(t, x) \in[0, T) \times \mathbb{R}^{d}, \\ u(T, x)=g(x) \quad ; \forall x \in \mathbb{R}^{d},\end{array}\right.$

where $D u=\left(\frac{\partial u_{i}}{\partial x_{j}}\right)_{i, j}$, with $i \in\{1, \ldots, n\}, j \in\{1, \ldots, d\}$, and Itô diffusion generator

$$
\mathcal{L}(t, x, u):=\left(\begin{array}{c}
L\left(t, x, u, D u_{1}, D^{2} u_{1}\right) \\
\vdots \\
L\left(t, x, u, D u_{n}, D^{2} u_{n}\right)
\end{array}\right)
$$

with $D u_{k}=\left(\frac{\partial u_{k}}{\partial x_{1}}, \ldots, \frac{\partial u_{k}}{\partial x_{d}}\right)^{\top}, D^{2} u_{k}=\left(\frac{\partial^{2} u_{k}}{\partial x_{i} \partial x_{j}}\right)_{i, j}$ and

$$
L(t, x, u, p, Q):=\frac{1}{2} \operatorname{tr}\left\{\sigma \sigma^{\top}(t, x, u) Q\right\}+\langle b(t, x, u, D u(t, x) \sigma(t, x, u)), p\rangle,
$$

for $p \in \mathbb{R}^{d}$ and $Q \in \mathbb{S}_{+}^{d}$. More precisely, if the FBSDEs (3) admits unique adapted solutions $\left(X^{t, x}, Y^{t, x}, Z^{t, x}\right)$ on each subintervals $[t, T] \subseteq[0, T]$ then

$$
Y_{s}^{t, x}=u\left(s, X_{s}^{t, x}\right), Z_{s}^{t, x}=D u\left(s, X_{s}^{t, x}\right) \sigma\left(s, X_{s}^{t, x}, Y_{s}^{t, x}\right) \quad ; \forall(s, x) \in[t, T] \times \mathbb{R}^{d} .
$$

Therefore, the function $u(t, x):=Y_{t}^{t, x}$ is a viscosity solution to the associated PDE (4) (see [21]). The relation $u(t, x)=Y_{t}^{t, x}$ is called the nonlinear Feynman-Kac formula.

From now on, we assume that $\sigma \sigma^{\top}$ is uniformly elliptic $i . e$. the diffusion matrices $\sigma \sigma^{\top}(t, x, y)$ are elliptic for all $(t, x, y) \in[0, T] \times \mathbb{R}^{d} \times \mathbb{R}^{n}$. In particular, when $h(t, x, y, z)=c(t, x) y+f(t, x)$ and $n=1$, the BSDE in (3) has explicit solution

$$
\begin{aligned}
Y_{s}^{t, x}= & \exp \left\{\int_{s}^{T} c\left(r, X_{r}^{t, x}\right) \mathrm{d} r\right\} g\left(X_{T}^{t, x}\right)+\int_{s}^{T} \exp \left\{\int_{s}^{r} c\left(\theta, X_{\theta}^{t, x}\right) d \theta\right\} f\left(r, X_{r}^{t, x}\right) \mathrm{d} r \\
& -\int_{s}^{T} \exp \left\{\int_{s}^{r} c\left(\theta, X_{\theta}^{t, x}\right) d \theta\right\} Z_{r}^{t, x} \mathrm{~d} W_{r} .
\end{aligned}
$$


In this case, the solution $u:[0, T] \times \mathbb{R}^{d} \rightarrow \mathbb{R}$ of the concerned PDE (4) can be expressed as $u(t, x)=Y_{t}^{t, x}=\mathbb{E}\left(Y_{t}^{t, x} \mid X_{t}=x\right)$, that is

$$
u(t, x)=\mathbb{E}\left[\exp \left\{\int_{t}^{T} c\left(r, X_{r}^{t, x}\right) \mathrm{d} r\right\} g\left(X_{T}^{t, x}\right)+\int_{t}^{T} \exp \left\{\int_{t}^{s} c\left(r, X_{r}^{t, x}\right) \mathrm{d} r\right\} f\left(s, X_{s}^{t, x}\right) \mathrm{d} s\right],
$$

which corresponds to the well-known Feynman-Kac formula (see e.g. [45, [56, 59]).

In the case of the $d$-dimensional viscous Burgers equation (from now on, we use $\nu / 2$ as viscosity instead of $\nu$ for the sake of simplicity)

$$
\left\{\begin{array}{l}
\frac{\partial u}{\partial t}+(u \cdot \nabla) u+\frac{\nu}{2} \Delta u+f=0 \quad ; 0 \leq t<T \\
\quad u(T)=g
\end{array}\right.
$$

where $f:[0, T] \times \mathbb{R}^{d} \rightarrow \mathbb{R}^{d}, g: \mathbb{R}^{d} \rightarrow \mathbb{R}^{d}$ and $\nu>0$, we can associate the coupled FBSDEs

$$
\left\{\begin{array}{l}
X_{s}^{t, x}=x+\int_{t}^{s} Y_{r}^{t, x} \mathrm{~d} r+\int_{t}^{s} \sqrt{\nu} \mathrm{d} W_{r} \\
Y_{s}^{t, x}=g\left(X_{T}^{t, x}\right)+\int_{s}^{T} f\left(r, X_{r}^{t, x}\right) \mathrm{d} r-\int_{s}^{T} \sqrt{\nu} Z_{r}^{t, x} \mathrm{~d} W_{r}
\end{array}\right.
$$

Then, $Y_{s}^{t, x}=u\left(s, X_{s}^{t, x}\right)$ and $Z_{s}^{t, x}=D u\left(s, X_{s}^{t, x}\right)$ for all $(s, x) \in[t, T] \times \mathbb{R}^{d}$ (see e.g. Theorem 4.1 in [43]). Alternatively, (5) admits the decoupled FBSDEs representation

$$
\left\{\begin{array}{l}
X_{s}^{t, x}=x+\int_{t}^{s} \sqrt{\nu} \mathrm{d} W_{r} \\
Y_{s}^{t, x}=g\left(X_{T}^{t, x}\right)+\int_{s}^{T}\left[f\left(r, X_{r}^{t, x}\right)+\frac{1}{\sqrt{\nu}} Z_{r}^{t, x} Y_{r}^{t, x}\right] \mathrm{d} r-\int_{s}^{T} \sqrt{\nu} Z_{r}^{t, x} \mathrm{~d} W_{r}
\end{array}\right.
$$

In the coupled FBSDEs (6) the process $Z^{t, x}$ can be removed by considering the conditional expectation $\mathbb{E}\left(\cdot \mid X_{t}^{t, x}=x\right)$, so that it is not necessary to compute it in order to estimate $Y^{t, x}$. This is an advantage compared to the decoupled representation (7) if we only are interested in the estimation of the process $Y_{s}^{t, x}$ without information about $Z_{s}^{t, x}$.

\subsection{The Incompressible Navier-Stokes equations}

From now on, by abuse of notation, we consider the backward Navier-Stokes equations for incompressible fluids in $\mathbb{R}^{d}$, for $d \in\{2,3\}$,

$$
\left\{\begin{array}{l}
\frac{\partial u}{\partial t}+(u \cdot \nabla) u+\frac{\nu}{2} \triangle u+\nabla p+f=0 \quad ; 0 \leq t<T \\
\nabla \cdot u=0, \quad u(T)=g
\end{array}\right.
$$

which is equivalent to the classical formulation (1) by a time-reversing transformation (still with $\nu / 2$ as viscosity). The Burgers equation (5) can be seen as a simplified version of the incompressible Navier-Stokes equations (8). Under regularity assumptions and supposing a divergence-free external force field $f$, an approach to incorporate both the pressure term $\nabla p$ and the incompressibility condition $\nabla \cdot u=0$ into the Burgers equation (5) is to consider the Poisson problem

$$
-\triangle p=\operatorname{div} \operatorname{div}(u \otimes u)
$$

where div $:=\nabla \cdot$ represents the divergence operator and $\otimes$ the tensor product (see e.g. [12, 44]). Then the incompressible Navier-Stokes equations system (8) is equivalent to

$$
\left\{\begin{array}{l}
\frac{\partial u}{\partial t}+\frac{\nu}{2} \triangle u+(u \cdot \nabla) u+\nabla(-\triangle)^{-1} \operatorname{div} \operatorname{div}(u \otimes u)+f=0 \quad ; 0 \leq t<T, \\
u(T)=g
\end{array}\right.
$$


F. Delbaen et al [26] introduced a coupled FBSDEs system (FBSDS) associated to (9) through the nonlinear Feynman-Kac formula $u(t, x)=Y_{t}^{t, x}$ and the probabilistic representation $\nabla p=\widetilde{Y}_{0}$ where $\widetilde{Y}$ is itself solution to a BSDE involving a Brownian motion $B$ independent from the one of the diffusion. The nonlocal operator $\nabla(-\triangle)^{-1}$ div div is represented by means of the BSDE defined on the infinite time interval $(0, \infty)$. Then, incorporating this extra BSDE to the FBSDEs (6) it is obtained a stochastic representation to the unsteady Navier-Stokes equations. More precisely, this new FBSDEs representation is

$$
\left\{\begin{array}{l}
d X_{s}^{t, x}=Y_{s}^{t, x} \mathrm{~d} s+\sqrt{\nu} \mathrm{d} W_{s} \quad ; s \in[t, T], \\
X_{t}^{t, x}=x, \\
-d Y_{s}^{t, x}=\left[f\left(s, X_{s}^{t, x}\right)+\widetilde{Y}_{0}\left(s, X_{s}^{t, x}\right)\right] \mathrm{d} s-\sqrt{\nu} Z_{s}^{t, x} \mathrm{~d} W_{s} \quad ; s \in[t, T], \\
Y_{T}^{t, x}=g\left(X_{T}^{t, x}\right), \\
-d \widetilde{Y}_{r}^{s, x}=\frac{27}{2 r^{3}} Y_{s}^{i} \cdot Y_{s}^{j}\left(s, x+B_{r}\right)\left(B_{r}-B_{\frac{2 r}{3}}\right)^{i}\left(B_{\frac{2 r}{3}}-B_{\frac{r}{3}}\right)^{j} B_{\frac{r}{3}} \mathrm{~d} r-\widetilde{Z}_{r}^{s, x} \mathrm{~d} B_{r} \quad ; r \in(0, \infty), \\
\tilde{Y}_{\infty}^{s, x}=0,
\end{array}\right.
$$

where $W$ and $B$ are two independent $d$-dimensional Brownian motions. Here $\widetilde{Y}_{0}\left(s, X_{s}^{t, x}\right)$ and $Y_{s}\left(s, x+B_{r}\right)$ means $\widetilde{Y}_{0}^{s, X_{s}^{t, x}}$ and $Y_{s}^{s, x+B_{r}}$, respectively.

In [26], the infinite interval $(0, \infty)$ of the probabilistic representation for the operator $\nabla(-\triangle)^{-1}$ div div is restricted to $\left[\frac{1}{N}, N\right]$, for $N \in(1, \infty)$. Hence the solution $u$ of $(9)$ on $\left(T_{0}, T\right]$, with $T_{0} \in[0, T)$, is approximated by $u^{N}$ which solves the PDE

$$
\left\{\begin{array}{l}
\frac{\partial u^{N}}{\partial t}+\frac{\nu}{2} \triangle u^{N}+\left(u^{N} \cdot \nabla\right) u^{N}+\mathbf{Q}^{N}\left(u^{N} \otimes u^{N}\right)+f=0 ; T_{0} \leq t<T, \\
u^{N}(T)=g
\end{array}\right.
$$

where for $N \in(1, \infty)$ and $\forall \phi, \psi \in H^{m}$, with $m>\frac{d}{2}+1$,

$$
\mathbf{Q}^{N}(\phi \otimes \psi)(x):=\mathbb{E} \int_{\frac{1}{N}}^{N} \frac{27}{2 s^{3}} \phi^{i} \cdot \psi^{j}\left(x+B_{s}\right)\left(B_{s}-B_{\frac{2 s}{3}}\right)^{i}\left(B_{\frac{2 s}{3}}-B_{\frac{s}{3}}\right)^{j} B_{\frac{s}{3}} \mathrm{~d} s ; \quad \forall x \in \mathbb{R}^{d} .
$$

Given $\nu>0, g \in H_{\sigma}^{m}$ and $f \in C\left([0, T] ; H_{\sigma}^{m-1}\right)$ with sufficient regularity $m>\frac{d}{2}+1$,

$$
\left\|u-u^{N}\right\|_{C\left([t, T] ; C^{k, \alpha}\right)} \leq \frac{K}{N^{\frac{\alpha}{4}}} ; \quad \forall t \in\left(T_{0}, T\right],
$$

for some $k \in \mathbb{Z}_{+}, \alpha \in(0,1)$ and $K>0$ independent of $N$ (see Theorem 6.6 in [26]). Then, the PDE (11) is associated through the nonlinear Feynman-Kac formula

$$
u^{N}(t, x)=Y_{t}^{t, x}
$$

to the following FBSDS

$$
\left\{\begin{array}{l}
d X_{s}^{t, x}=Y_{s}\left(s, X_{s}^{t, x}\right) \mathrm{d} s+\sqrt{\nu} \mathrm{d} W_{s} \quad ; s \in[t, T] \\
X_{t}^{t, x}=x \\
-d Y_{s}\left(s, X_{s}^{t, x}\right)=\left[f\left(s, X_{s}^{t, x}\right)+\mathbf{Q}^{N}\left(Y_{s} \otimes Y_{s}\right)\left(s, X_{s}^{t, x}\right)\right] \mathrm{d} s-\sqrt{\nu} Z_{s}^{t, x} \mathrm{~d} W_{s} \quad ; s \in[t, T], \\
Y_{T}(T, x)=g(x), \\
\mathbf{Q}^{N}\left(Y_{s} \otimes Y_{s}\right)(s, x)=\mathbb{E} \int_{\frac{1}{N}}^{N} \frac{27}{2 r^{3}} Y_{s}^{i} \cdot Y_{s}^{j}\left(s, x+B_{r}\right)\left(B_{r}-B_{\frac{2 r}{3}}\right)^{i}\left(B_{\frac{2 r}{3}}-B_{\frac{r}{3}}\right)^{j} B_{\frac{r}{3}} \mathrm{~d} r,
\end{array}\right.
$$

where $W$ and $B$ are independent $d$-dimensional Wiener processes and, by abuse of notation, we write $Y_{s}(t, y):=Y_{s}^{t, y}$. Moreover, it is worth pointing out that $Y_{s}^{t, \cdot}=Y_{s}^{s, X_{s}^{t, *}}$ (see Theorem 6.4 and Remark 6.2 in [26]). 


\section{$3 \quad$ Numerical methodology}

In this section, we specify some probabilistic algorithms for the numerical solution of FBSDEs. The stochastic representations for the solutions of the Burgers equation and the incompressible Navier-Stokes equations follow from systems (6) and (10), respectively. In the first case, we consider the approach by F. Delarue and S. Menozzi [24, 25, and then we incorporate quantization as a control variate variable to reduce the variance of the Monte Carlo estimation of the expected values. Then, we study the incompressible Navier-Stokes equations. To this end, we consider the numerical algorithm introduced by F. Delbaen et al [26] and then we propose a novel probabilistic algorithm by incorporating the numerical treatment of the pressure term. The rest of the section is devoted to the probabilistic tools used in the numerical algorithms.

We begin with some preliminaries to the numerical approximation of FBSDEs. More precisely, let $T>0$ be fixed and given $N_{T} \in \mathbb{Z}_{+}$consider a regular mesh of $[0, T]$ with time-step $h:=T / N_{T}$ i.e. set the temporal nodes $[0, T] \cap \mathcal{T}_{h}$, with $\mathcal{T}_{h}:=h \cdot \mathbb{Z}$, or well

$$
t_{k}:=k \cdot h ; \quad \forall k \in\left\{0, \ldots, N_{T}\right\},
$$

and the infinite Cartesian grid $\mathcal{C}_{\delta}:=\delta \cdot \mathbb{Z}^{d}$ of uniform spatial-step $\delta>0$. Moreover, let $\Pi_{\delta}$ be the projection mapping on the grid $\mathcal{C}_{\delta}$ defined by

$$
\Pi_{\delta}(x):=\arg \min \left\{\|x-\bar{x}\|: \bar{x} \in \mathcal{C}_{\delta}\right\} ; \quad \forall x \in \mathbb{R}^{d} .
$$

Observe that for $\alpha$-Hölder continuous functions $F: \mathbb{R}^{d} \rightarrow \mathbb{R}^{d}$ the projection error results

$$
\left\|F(x)-F\left(\Pi_{\delta}(x)\right)\right\| \leq K \delta^{\alpha} .
$$

From now on, we consider sufficient small discretization parameters of time $h>0$ and space $\delta>0$ such that $\delta<h$ (see Subsection 3.1).

For all $k \in\left\{0, \ldots, N_{T}-1\right\}$, we denote $\Delta W_{k}=W_{t_{k+1}}-W_{t_{k}}$ and refer as $q\left(\Delta W_{k}\right)=$ $\sqrt{h} q\left(\frac{\Delta W_{k}}{\sqrt{h}}\right)$ to the optimal quantization of the underlying Gaussian variable $\Delta W_{k}$. Similarly, $q\left(W_{s}\right)=\sqrt{s} q\left(\frac{W_{s}}{\sqrt{s}}\right)$ (see Subsection 3.3 below).

\subsection{Burgers equation}

As a first point, since the Burgers equation representation (6) we are interested in systems of FBSDEs (3) whose drift terms $b$ and $h$ are independent of the diffusion coefficient $Z$ i.e. FBSDEs of the form

$$
\forall s \in[t, T],\left\{\begin{array}{l}
X_{s}=x+\int_{t}^{s} b\left(r, X_{r}, Y_{r}\right) \mathrm{d} r+\int_{t}^{s} \sigma\left(r, X_{r}, Y_{r}\right) \mathrm{d} W_{r}, \\
Y_{s}=g\left(X_{T}\right)+\int_{s}^{T} h\left(r, X_{r}, Y_{r}\right) \mathrm{d} r-\int_{s}^{T} Z_{r} \mathrm{~d} W_{r} .
\end{array}\right.
$$

As usual, we can remove the martingale part of the BSDE by taking the conditional expectation $\mathbb{E}\left(\cdot \mid X_{t}=x\right)$ and so the dependence of $Y$ on the process $Z$. Thus we focus on the numerical solution of the process $Y$ and, since the nonlinear Feynman-Kac formula, the estimation of the strong solution $u$ of the related PDE (4) by means of a probabilistic numerical algorithm.

Usually the numerical schemes for FBSDEs involve backward regressions to compute estimations of the processes $(X, Y, Z$ ) (see e.g. [43, 49]). The following numerical scheme $\bar{u}$ approximates the process $Y$ involved in the FBSDEs (14).

Algorithm 3.1 (F. Delarue and S. Menozzi [24]). Define

$$
\bar{u}(T, x)=g(x) ; \quad \forall x \in \mathcal{C}_{\delta} .
$$


For each temporal node $t_{k}$ with $k \in\left\{0, \ldots, N_{T}-1\right\}$ and any spatial position $x \in \mathcal{C}_{\delta}$, compute recursively

$$
\begin{aligned}
\mathcal{T}\left(t_{k}, x\right) & =b\left(t_{k+1}, x, \bar{u}\left(t_{k+1}, x\right)\right) \cdot h+\sigma\left(t_{k+1}, x, \bar{u}\left(t_{k+1}, x\right)\right) q\left(\Delta W_{k}\right) \\
\bar{u}\left(t_{k}, x\right) & =\mathbb{E}\left[\bar{u}\left(t_{k+1}, \Pi_{\delta}\left(x+\mathcal{T}\left(t_{k}, x\right)\right)\right)\right]+h\left(t_{k+1}, x, \bar{u}\left(t_{k+1}, x\right)\right) \cdot h .
\end{aligned}
$$

The process $\mathcal{T}\left(t_{k}, x\right)$ corresponds to the Euler-Maruyama scheme applied to the Itô SDE. Its convergence is well known in a Hölder continuous setting when the quantization of $\Delta W_{k}$ is not considered (see [48]). Quantizing the underlying Gaussian variables $q\left(\Delta W_{k}\right)$ provides us with an efficient method to deal with the expectation approximations to the terms $\bar{u}\left(t_{k}, x\right)$, instead of the classical Monte Carlo procedure. By its way the projection operator $\Pi_{\delta}$ onto the spatial grid incorporates an extra source of error to the order of convergence of the numerical algorithm. The spatial discretization $\delta>0$ must be smaller than the time step $h>0$ to capture the dynamics of the drift and diffusion parts of $\mathcal{T}\left(t_{k}, x\right)$ in the computation of $\Pi_{\delta}\left(x+\mathcal{T}\left(t_{k}, x\right)\right.$ ). Indeed, the discretization $\mathcal{T}^{N}\left(t_{k}, x\right)$ has drift term of order $h$ and diffusion part of order $\sqrt{h}$. Hence the spatial mesh has to be fine enough to capture the local influence of $x+\mathcal{T}^{N}\left(t_{k}, x\right)$ by means of the projector $\Pi_{\delta}$.

All this elements are bounded to the computation of Algorithm 3.1] (see Section 4 of [24] and 25] for details and convergence).

We incorporate quantization as a control variate variable to the Monte Carlo computation of mean values (see Subsection 3.3 for details). More precisely, we introduce the following numerical scheme:

Algorithm 3.2. Define

$$
\bar{u}(T, x)=g(x) ; \quad \forall x \in \mathcal{C}_{\delta} .
$$

For each time node $k \in\left\{0, \ldots, N_{T}-1\right\}$ and any position $x \in \mathcal{C}_{\delta}$, compute

$$
\begin{aligned}
\mathcal{T}\left(t_{k}, x\right) & =b\left(t_{k+1}, x, \bar{u}\left(t_{k+1}, x\right)\right) \cdot h+\sigma\left(t_{k+1}, x, \bar{u}\left(t_{k+1}, x\right)\right) q\left(\Delta W_{k}\right), \\
\bar{u}\left(t_{k}, x\right) & =\mathbb{E}\left[\bar{u}\left(t_{k+1}, \Pi_{\delta}\left(x+\mathcal{T}\left(t_{k}, x\right)\right)\right)\right]+h\left(t_{k+1}, x, \bar{u}\left(t_{k+1}, x\right)\right) \cdot h \\
& +\frac{1}{M} \sum_{l=1}^{M}\left[\bar{u}\left(t_{k+1}, \Pi_{\delta}\left(x+\mathcal{T}^{(l)}\left(t_{k}, x\right)\right)\right)-\bar{u}\left(t_{k+1}, \Pi_{\delta}\left(x+\widehat{\mathcal{T}}^{(l)}\left(t_{k}, x\right)\right)\right)\right] .
\end{aligned}
$$

Here $\Delta W_{k}^{(l)}=W_{t_{k+1}}^{(l)}-W_{t_{k}}^{(l)}$ are $M \in \mathbb{Z}_{+}$independent realizations of the Gaussian variable $\Delta W_{k}$. Moreover, $\mathcal{T}^{(l)}\left(t_{k}, x\right):=b\left(t_{k+1}, x, \bar{u}\left(t_{k+1}, x\right)\right) \cdot h+\sigma\left(t_{k+1}, x, \bar{u}\left(t_{k+1}, x\right)\right) \Delta W_{k}^{(l)}$ and $\widehat{\mathcal{T}}^{(l)}\left(t_{k}, x\right):=b\left(t_{k+1}, x, \bar{u}\left(t_{k+1}, x\right)\right) \cdot h+\sigma\left(t_{k+1}, x, \bar{u}\left(t_{k+1}, x\right)\right) q\left(\Delta W_{k}^{(l)}\right)$.

Note that the convergence of Algorithm 3.1 involves the optimality of quantization parameters to the underlying Gaussian random variables. However, the quantization as control variates to the Monte Carlo method can reduce the approximation error or improve the computational effort to the calculation of the conditional expectations. In [46], Algorithm 3.2 have been tested with success to the Burgers equation in some numerical tests proposed by [24, 25].

\subsection{Incompressible Navier-Stokes equations}

Delbaen et al [26] propose a numerical algorithm $\bar{u}^{N}$ to approximate the process $Y$ that solves (13) and then the solution $u^{N}$ of (11). We present now the general algorithm which computes a numerical approximation $\bar{u}^{N}$ of the velocity field $u$ solution to the incompressible Navier-Stokes equation (8). 


$$
\bar{u}^{N}(T, x)=g(x) ; \quad \forall x \in \mathcal{C}_{\delta} .
$$

Then for each $k \in\left\{0, \ldots, N_{T}-1\right\}$ and all $x \in \mathcal{C}_{\delta}$, compute

$$
\begin{aligned}
\mathcal{T}^{N}\left(t_{k}, x\right) & =\bar{u}^{N}\left(t_{k+1}, x\right) \cdot h+\sqrt{\nu} q\left(\Delta W_{k}\right) \\
Q^{N}\left(t_{k+1}, x\right) & =\mathbb{E} \int_{\frac{1}{3 N}}^{\frac{N}{3}} \frac{3}{2 r^{3}} \bar{u}_{i}^{N} \cdot \bar{u}_{j}^{N}\left(t_{k+1}, \Pi_{\delta}\left(x+q\left(\bar{B}_{r}\right)+q\left(\widetilde{B}_{r}\right)+q\left(\widehat{B}_{r}\right)\right)\right) q^{i}\left(\bar{B}_{r}\right) q^{j}\left(\widetilde{B}_{r}\right) q\left(\widehat{B}_{r}\right) \mathrm{d} r \\
\bar{u}^{N}\left(t_{k}, x\right) & =\mathbb{E}\left[\bar{u}^{N}\left(t_{k+1}, \Pi_{\delta}\left(x+\mathcal{T}^{N}\left(t_{k}, x\right)\right)\right)\right]+\left(f\left(t_{k+1}, x\right)+Q^{N}\left(t_{k+1}, x\right)\right) \cdot h .
\end{aligned}
$$

For each time $t_{k} \in[0, T]$, Algorithm 3.3 computes the approximation $\bar{u}^{N}\left(t_{k}, \cdot\right)$ of $u^{N}\left(t_{k}, \cdot\right)$ over the spatial grid, where $u^{N}$ solves the backward Navier-Stokes equation (9). Therefore the velocity field $u\left(t_{k}, \cdot\right)$ of the incompressible Navier-Stokes equations (1) is approximated by $-\bar{u}^{N}\left(T-t_{k}, \cdot\right)$.

As is the Burgers equation context, the spatial step $\delta>0$ must be smaller than the time step $h>0$. Moreover, the probabilistic representation of the gradient pressure involves perturbations of order $\sqrt{r}$ to each particle position $x$. It would be necessary to have spatial discretizations $\delta<\frac{1}{\sqrt{N}}$ to capture its dynamics by means of the projector $\Pi_{\delta}$.

Given the vector field $\bar{u}^{N}\left(t_{k+1}, \cdot\right) \approx Y_{t_{k+1}}^{t_{k+1},}$, the algorithm constructs the approximation $\bar{u}^{N}\left(t_{k}, x\right)$ of $Y_{t_{k}}\left(t_{k}, x\right)$ for all $x \in \mathcal{C}_{\delta}$, with $Y$ solving (13). This procedure involves the local time integration

$$
\begin{aligned}
\int_{t_{k}}^{t_{k+1}} \mathrm{Q}^{N}\left(Y_{s} \otimes Y_{s}\right)\left(s, X_{s}^{t_{k}, x}\right) \mathrm{d} s & \approx \mathbb{E} \int_{\frac{1}{N}}^{N} \frac{27}{2 r^{3}} Y_{t_{k+1}}^{i} \cdot Y_{t_{k+1}}^{j}\left(t_{k+1}, x+B_{r}\right)\left(B_{r}-B_{\frac{2 r}{3}}\right)^{i}\left(B_{\frac{2 r}{3}}-B_{\frac{r}{3}}\right)^{j} B_{\frac{r}{3}} \mathrm{~d} r \cdot r \\
& \approx \mathbb{E} \int_{\frac{1}{N}}^{N} \frac{27}{2 r^{3}} \bar{u}_{i}^{N} \cdot \bar{u}_{j}^{N}\left(t_{k+1}, x+B_{r}\right)\left(B_{r}-B_{\frac{2 r}{3}}\right)^{i}\left(B_{\frac{2 r}{3}}-B_{\frac{r}{3}}\right)^{j} B_{\frac{r}{3}} \mathrm{~d} r \cdot h \\
& =\mathbb{E} \int_{\frac{1}{3 N}}^{\frac{N}{3}} \frac{3}{2 r^{3}} \bar{u}_{i}^{N} \cdot \bar{u}_{j}^{N}\left(t_{k+1}, x+\bar{B}_{r}+\widetilde{B}_{r}+\widehat{B}_{r}\right) \bar{B}_{r}^{i} \widetilde{B}_{r}^{j} \widehat{B}_{r} \mathrm{~d} r \cdot h \\
& \approx \mathbb{E} \int_{\frac{1}{3 N}}^{\frac{N}{3}} \frac{3}{2 r^{3}} \bar{u}_{i}^{N} \cdot \bar{u}_{j}^{N}\left(t_{k+1}, \Pi_{\delta}\left(x+\bar{B}_{r}+\widetilde{B}_{r}+\widehat{B}_{r}\right)\right) \bar{B}_{r}^{i} \widetilde{B}_{r}^{j} \widehat{B}_{r} \mathrm{~d} r \cdot h .
\end{aligned}
$$

Here the increments of $B$ are replaced by three independent $d$-dimensional Brownian motions $\bar{B}, \widetilde{B}$ and $\widehat{B}$ independent of $W$. Then, involving the quantization of the underlying Gaussian random variables we obtain

$$
\int_{t_{k}}^{t_{k+1}} \mathbf{Q}^{N}\left(Y_{s} \otimes Y_{s}\right)\left(s, X_{s}^{t_{k}, x}\right) \mathrm{d} s \approx Q^{N}\left(t_{k+1}, x\right) \cdot h .
$$

Computing expectations of integrals depending on paths of Brownian motions introduces an additional source of error in the numerical treatment of the pressure term. We then show how to efficiently compute $\mathcal{Q}^{N}$ in Algorithm 3.3 so that $\bar{u}^{N}$ approximates $u^{N}$ given by (11) (see Subsection 3.4).

The quantization of the underlying Gaussian variables permits us to compute mean values easily, with its estimation error depending on the dimension $d$ and the number of quantization points. As in the Burgers equation, we can use quantization as a control variate to the estimation of the pressure term, but increasing considerably the computational effort of the algorithm especially when $d=3$. Hence, a convenient variance reduction technique needs to be considered to deal with path-dependent functionals of Brownian motion. We mention for example variance reduction techniques in [41, 39]. 


\subsection{Estimation of conditional expectations}

Suppose that $U: \Omega \rightarrow \mathbb{R}^{m}$ is a square integrable random variable and $F: \mathbb{R}^{m} \rightarrow \mathbb{R}^{d}$ is uniformly $\alpha$-Hölder continuous with Hölder exponent $\alpha \in(0,1]$, such that $F(U) \in L_{\mathcal{F}}^{2}\left(\Omega ; \mathbb{R}^{d}\right)$. The usual way to estimate expectations is by means of the Monte Carlo approximation

$$
\mathbb{E}[F(U)] \approx \frac{1}{M} \sum_{i=1}^{M} F\left(U^{(i)}\right),
$$

where $U^{(i)}$ are $M$ independent realizations of the underlying random variable $U$. From now on, the averages are computed in a component-wise manner. It is well-known that the Monte Carlo method (16) converges in law to the mean $\mathbb{E}[F(U)]$ with estimation error depending on $\sqrt{\operatorname{Var} F(U)} / \sqrt{M}$ as $M$ tends to $\infty$.

We use the quantization method to estimate mean values. Indeed, let $q(U)$ be a quantizer of $U$ i.e. an approximation of $U$ by a discrete random variable $q(U)$ taking values in a finite set $\Gamma=\left\{v_{1}, \ldots, v_{Q}\right\} \subset \mathbb{R}^{m}$. Suppose that $q(U)=\sum_{i=1}^{Q} v_{i} \mathbf{1}_{C_{i}}(U)$ where $C=\left\{C_{1}, \ldots, C_{Q}\right\}$ is a partition of $\mathbb{R}^{m}$ associated to $\Gamma$ be means of $v_{i} \in C_{i}$ for all $i \in\{1, \ldots, Q\}$. Then

$$
\mathbb{E}[F(U)] \approx \mathbb{E}[F(q(U))]=\sum_{i=1}^{Q} \mathbb{P}\left(U \in C_{i}\right) \cdot F\left(v_{i}\right) .
$$

Since $F$ is $\alpha$-Hölder continuous, Jensen's inequality implies that the error of estimation is bounded by

$$
\|\mathbb{E}[F(U)]-\mathbb{E}[F(q(U))]\| \leq K\left(\mathbb{E}\left[\|U-q(U)\|^{2}\right]\right)^{\alpha / 2}
$$

where the quantization error in the $L^{2}$-norm (to the power 2 ) is given by

$$
\|U-q(U)\|_{2}^{2}=\sum_{i=1}^{Q} \mathbb{E}\left[\left\|U-v_{i}\right\|^{2} \mathbf{1}_{C_{i}}(U)\right] .
$$

Thus, the key point is to provide convenient sets $\Gamma$ and $C$ such that the quadratic quantization error is optimal. An optimal quantizer is found through a stochastic gradient procedure over the positions of the points $v_{1}, \ldots, v_{Q}$. The cells $C_{i}$ are then given by a Voronoï partition of the space. Hence, for a given realization $u \in \mathbb{R}^{m}$ of $U$, we set

$$
q(u):=v_{i} \text { such that }\left\|u-v_{i}\right\| \leq\left\|u-v_{j}\right\| ; \forall j=1, \ldots, Q, j \neq i .
$$

In other words, $q(u)$ is the closest point to $u$ among the $v_{i}$ 's. We refer to [34, 54, 20, 19] for details on optimal quantization. In particular, the case of a $m$-dimensional Gaussian random variable $\xi \sim \mathcal{N}\left(0, I_{m}\right)$ is is treated in details in [54].

A consequence from the Zador theorem [34] is that

$$
\mathbb{E}\left[\|\xi-q(\xi)\|^{2}\right]^{1 / 2} \leq K(m) Q^{-1 / m}
$$

where $K(m)$ is a constant which depends from the dimension $m$. Hence we have a nonasymptotic error bound

$$
\|\mathbb{E}[F(U)]-\mathbb{E}[F(q(U))]\| \leq \frac{K}{Q^{\alpha / m}},
$$

depending on the dimension $m$ and the number $Q$ of quantizer points. We see that quantization is better in low dimension. 
Tables for optimal quantizers are provided in the web site [20], which we use for our numerical implementations ${ }^{1}$. Thus, given an application $q$ which gives the optimal quantizer for $\xi \sim \mathcal{N}\left(0, I_{m}\right)$, we set

$$
q(\zeta):=\mu+\Sigma q\left(\Sigma^{-1}(\zeta-\mu)\right) \text { for } \zeta \sim \mathcal{N}\left(\mu, \Sigma \Sigma^{T}\right)
$$

Quantization is also a convenient tool for constructing a control variate in order to perform a variance reduction technique in Monte Carlo simulations (see e.g. [30] for an introduction to control variates). More precisely, we use

$$
\mathbb{E}[F(U)] \approx \mathbb{E}[F(q(U))]+\frac{1}{M} \sum_{i=1}^{M}\left[F\left(U^{(i)}\right)-F\left(q\left(U^{(i)}\right)\right)\right] .
$$

The error of the estimation $(18)$ is then $\sqrt{\operatorname{Var}(F(U)-F(q(U)))} / \sqrt{M}$ as $M$ goes to $\infty$. Thus, the error is reduced when $q(U)$ is close enough to $U$. The order $M^{-1 / 2} Q^{-1 / m}$ is obtained for the Gaussian case. We refer to [55, 41, 19] for reduction variance techniques that use quantization.

As a special case, let $U^{1}, \ldots, U^{\ell}$ be $\ell$ independent and identically distributed square integrable random variables $U^{k}: \Omega \rightarrow \mathbb{R}^{m}$ and $F:\left(\mathbb{R}^{m}\right)^{\ell} \rightarrow \mathbb{R}^{d}$ an $\alpha$-Hölder continuous function such that $F\left(U^{1}, \ldots, U^{\ell}\right)$ belongs to $L_{\mathcal{F}}^{2}\left(\Omega ; \mathbb{R}^{d}\right)$. Then, we compute

$$
\begin{aligned}
\mathbb{E}\left[F\left(U^{1}, \ldots, U^{\ell}\right)\right] & \approx \mathbb{E}\left[F\left(q\left(U^{1}\right), \ldots, q\left(U^{\ell}\right)\right)\right] \\
& =\sum_{i_{1}, \ldots, i_{l}=1}^{Q} \mathbb{P}\left(U^{1} \in C_{i_{1}}\right) \times \cdots \times \mathbb{P}\left(U^{\ell} \in C_{i_{l}}\right) F\left(v_{i_{1}}, \ldots, v_{i_{l}}\right)
\end{aligned}
$$

because $U^{1}, \ldots, U^{\ell}$ are independent. The same quantizers points are taken for each random variable.

\subsection{Integrals involving paths of Brownian motions}

In the FBSDS (10) the pressure term is represented by

$$
\nabla p\left(s, X_{s}^{t, x}\right)=\tilde{Y}_{0}\left(s, X_{s}^{t, x}\right) .
$$

Then the approximated solution $u^{N}$ is estimated by means of the stochastic process $Y$ of the truncated FBSDS (13) and involves its approximation $\mathbf{Q}^{N}\left(Y_{s} \otimes Y_{s}\right)\left(s, X_{s}^{t, x}\right)$, for $N \in \mathbb{Z}_{+}$. Given the estimations $\bar{u}^{N}\left(t_{k+1}, x\right)$ of $Y_{t_{k+1}}\left(t_{k+1}, x\right)$ for all $x \in \mathcal{C}_{\delta}$, we approximate $Y_{t_{k}}\left(t_{k}, x\right)$ over the spatial grid by locally integrating the BSDE that defines $\left(Y_{s}^{t_{k}, x}\right)_{s \in\left[t_{k}, t_{k+1}\right]}$. Concerning to the gradient of the pressure, for each $k \in\left\{0, \ldots, N_{T}-1\right\}$ and $x \in \mathcal{C}_{\delta}$,

$\mathbb{E} \int_{t_{k}}^{t_{k+1}} \mathbf{Q}^{N}\left(Y_{s} \otimes Y_{s}\right)\left(s, X_{s}^{t, x}\right) d s \approx \mathbb{E} \int_{\frac{1}{N}}^{N} \frac{27}{2 r^{3}} \bar{u}_{i}^{N} \cdot \bar{u}_{j}^{N}\left(t_{k+1}, x+B_{r}\right)\left(B_{r}-B_{\frac{2 r}{3}}\right)^{i}\left(B_{\frac{2 r}{3}}-B_{\frac{r}{3}}\right)^{j} B_{\frac{r}{3}} \mathrm{~d} r \cdot h$.

In [26], the increments of $B$ are replaced by three independent $d$-dimensional Brownian motions $\bar{B}, \widetilde{B}$ and $\widehat{B}$ independent of $W$. This procedure involves the local time integration

$$
\begin{aligned}
\int_{t_{k}}^{t_{k+1}} \mathrm{Q}^{N}\left(Y_{s} \otimes Y_{s}\right)\left(s, X_{s}^{t_{k}, x}\right) d s & \approx \mathbb{E} \int_{\frac{1}{N}}^{N} \frac{27}{2 r^{3}} \bar{u}_{i}^{N} \cdot \bar{u}_{j}^{N}\left(t_{k+1}, x+B_{r}\right)\left(B_{r}-B_{\frac{2 r}{3}}\right)^{i}\left(B_{\frac{2 r}{3}}-B_{\frac{r}{3}}\right)^{j} B_{\frac{r}{3}} \mathrm{~d} r \cdot h \\
& =\mathbb{E} \int_{\frac{1}{3 N}}^{\frac{N}{3}} \frac{3}{2 r^{3}} \bar{u}_{i}^{N} \cdot \bar{u}_{j}^{N}\left(t_{k+1}, x+\bar{B}_{r}+\widetilde{B}_{r}+\widehat{B}_{r}\right) \bar{B}_{r}^{i} \widetilde{B}_{r}^{j} \widehat{B}_{r} \mathrm{~d} r \cdot h \\
& \approx \mathbb{E} \int_{\frac{1}{3 N}}^{\frac{N}{3}} \frac{3}{2 r^{3}} \bar{u}_{i}^{N} \cdot \bar{u}_{j}^{N}\left(t_{k+1}, \Pi_{\delta}\left(x+\bar{B}_{r}+\widetilde{B}_{r}+\widehat{B}_{r}\right)\right) \bar{B}_{r}^{i} \widetilde{B}_{r}^{j} \widehat{B}_{r} \mathrm{~d} r \cdot h .
\end{aligned}
$$

\footnotetext{
${ }^{1}$ There exists several ways to consider the quantization of a vector: we could quantize each components, the vector as a whole, or sub-vectors.
} 
Hence denoting

$$
F\left(r, \bar{B}_{r}, \widetilde{B}_{r}, \widehat{B}_{r}\right):=\frac{3}{2 r^{3}} \bar{u}_{i}^{N} \cdot \bar{u}_{j}^{N}\left(t_{k+1}, \Pi_{\delta}\left(x+\bar{B}_{r}+\widetilde{B}_{r}+\widehat{B}_{r}\right)\right) \bar{B}_{r}^{i} \widetilde{B}_{r}^{j} \widehat{B}_{r}
$$

we aim at computing numerically the term

$$
Q^{N}\left(t_{k+1}, x\right)=\mathbb{E} \int_{\frac{1}{3 N}}^{\frac{N}{3}} F\left(r, \bar{B}_{r}, \widetilde{B}_{r}, \widehat{B}_{r},\right) d r
$$

for $\bar{u}^{N}=\left(\bar{u}_{1}^{N}, \ldots, \bar{u}_{d}^{N}\right)^{\top}$ and three independent $d$-dimensional standard Brownian motions. The size of the interval $\left[\frac{1}{3 N}, \frac{N}{3}\right]$ determines the quality of the approximation in 12 so that accurate estimations are obtained for large values of $N$, leading to costly Monte Carlo approximations.

A first intent to compute the term $Q^{N}$ is to use the quantization of the underlying Gaussian processes together with Riemann sum approximations to the integrals. Indeed, let $N_{R} \in \mathbb{Z}_{+}$ and define an uniform time step $\tau:=\left(\frac{N}{3}-\frac{1}{3 N}\right) / N_{R}$, with equidistant nodes

$$
s_{\ell}:=\frac{1}{3 N}+(\ell-1) \cdot \tau ; \quad \forall \ell \in\left\{1, \ldots, N_{R}\right\}
$$

Therefore, denoting by

$$
\bar{\xi}_{r}:=\frac{\bar{B}_{r}}{\sqrt{r}}, \widetilde{\xi}_{r}:=\frac{\widetilde{B}_{r}}{\sqrt{r}}, \text { and } \widehat{\xi}_{r}:=\frac{\widehat{B}_{r}}{\sqrt{r}},
$$

the pressure gradient term is estimated from $Q^{N}\left(t_{k+1}, x\right) \approx \widehat{Q}^{N}\left(t_{k+1}, x\right)$ with

$$
\begin{array}{r}
\widehat{Q}^{N}\left(t_{k+1}, x\right):=\mathbb{E} \sum_{\ell=1}^{N_{R}} \frac{3}{2\left(\sqrt{s_{\ell}}\right)^{3}} \bar{u}_{i}^{N} \cdot \bar{u}_{j}^{N}\left(t_{k+1}, \Pi_{\delta}\left(x+\sqrt{s_{\ell}}\left[q\left(\bar{\xi}_{s_{\ell}}\right)+q\left(\widetilde{\xi}_{s_{\ell}}\right)+q\left(\widehat{\xi}_{s_{\ell}}\right)\right]\right)\right) \\
\times q^{i}\left(\bar{\xi}_{s_{\ell}}\right) q^{j}\left(\widetilde{\xi}_{s_{\ell}}\right) q\left(\widehat{\xi}_{s_{\ell}}\right) \cdot \tau .
\end{array}
$$

Remark 1 . In case of $\alpha$-Hölder continuous function $\phi: \mathbb{R}^{d} \rightarrow \mathbb{R}^{d}$ we consider

$$
F\left(r, \bar{B}_{r}, \widetilde{B}_{r}, \widehat{B}_{r}\right):=\frac{3}{2 r^{3}} \phi_{i} \cdot \phi_{j}\left(\Pi_{\delta}\left(x+\bar{B}_{r}+\widetilde{B}_{r}+\widehat{B}_{r}\right)\right) \bar{B}_{r}^{i} \widetilde{B}_{r}^{j} \widehat{B}_{r}
$$

and take

$$
\begin{aligned}
\mathbb{E} \int_{\frac{1}{3 N}}^{\frac{N}{3}} F\left(r, \bar{B}_{r}, \widetilde{B}_{r}, \widehat{B}_{r},\right) \mathrm{d} r & \approx \mathbb{E} \sum_{\ell=1}^{N_{R}} F\left(s_{\ell}, \bar{B}_{s_{\ell}}, \widetilde{B}_{s_{\ell}}, \widehat{B}_{s_{\ell}}\right) \cdot \tau \\
& \approx \mathbb{E} \sum_{\ell=1}^{N_{R}} F\left(s_{\ell}, q\left(\bar{B}_{s_{\ell}}\right), q\left(\widetilde{B}_{s_{\ell}}\right), q\left(\widehat{B}_{s_{\ell}}\right)\right) \cdot \tau
\end{aligned}
$$

for all $x \in \mathcal{C}_{\delta}$, which involves simulating $N_{R} \times 3 \times d$ Gaussian random variables. The error of estimation depends on $d \in\{2,3\}, \alpha \in(0,1)$, and the discretization parameters $\tau>0$ and $Q \in \mathbb{Z}_{+}$.

\subsection{Computer implementation}

The velocity field $u$ that solves the Navier-Stokes equations system (1) is estimated by

$$
u\left(t_{k}, x\right) \approx-\bar{u}^{N}\left(T-t_{k}, x\right) ; \quad \forall\left(t_{k}, x\right) \in[0, T] \cap \mathcal{T}_{h} \times[0,2 \pi]^{d} \cap \mathcal{C}_{\delta} .
$$


To do this, we initialize

$$
\bar{u}^{N}(T, x)=-u(0, x) ; \quad \forall x \in[0,2 \pi]^{d} \cap \mathcal{C}_{\delta} .
$$

Then for each $k$ from $N_{T}-1$ to 0 , we compute

$$
\bar{u}^{N}\left(t_{k}, x\right) ; \quad \forall x \in[0,2 \pi]^{d} \cap \mathcal{C}_{\delta},
$$

from the velocity field $\bar{u}^{N}\left(t_{k+1}, \cdot\right)$ and the optimal quantizers for standard Gaussian random variables $\mathcal{N}\left(0, I_{d}\right)$. In this procedure, let $\widehat{\Pi}_{\delta}$ be the projection mapping on the computational grid $[0,2 \pi]^{d} \cap \mathcal{C}_{\delta}$ defined by

$$
\widehat{\Pi}_{\delta}(x):=\arg \min \left\{\left\|\left(x-2 \pi \cdot\left\lfloor\frac{x}{2 \pi}\right\rfloor\right)-\bar{x}\right\|: \bar{x} \in[0,2 \pi]^{d} \cap \mathcal{C}_{\delta}\right\} ; \quad \forall x \in \mathbb{R}^{d} .
$$

Here \lfloor\rfloor denotes the floor function. The approximation $\bar{u}^{N}$ is extended by periodicity from $[0,2 \pi]^{d} \cap \mathcal{C}_{\delta}$ to the complete spatial grid $\mathcal{C}_{\delta}$.

More precisely, we present the following algorithm:

Algorithm 3.4. (1) Define the dimension $d \in\{2,3\}$, a final time $T>0$ and the viscosity parameter $\nu>0$. Fix the truncation parameter $N \in \mathbb{N}$, time-step $h=\frac{T}{N_{T}}$; with $N_{T} \in \mathbb{N}$, and spatial-step $\delta=\frac{2 \pi}{N_{\delta}}>0$, with $N_{\delta} \in \mathbb{N}$. Define the initial velocity field $g(x)$ and the external force $f(t, x)$ for each $t \in[0, T] \cap \mathcal{T}_{h}$ and for all $x \in[0,2 \pi]^{d} \cap \mathcal{C}_{\delta}$. Consider $Q \in \mathbb{N}$ grid points for quantization, and $N_{R} \in \mathbb{N}$ subintervals of $\left[\frac{1}{3 N}, \frac{N}{3}\right]$ with step size $\tau=\left(\frac{N}{3}-\frac{1}{3 N}\right) / N_{R}$.

(2) Initialize

$$
\bar{u}^{N}(T, x)=-g(x) ; \quad \forall x \in[0,2 \pi]^{d} \cap \mathcal{C}_{\delta} .
$$

(3) For each $k$ from $N_{T}-1$ to 0 , do for all $x \in[0,2 \pi]^{d} \cap \mathcal{C}_{\delta}$ :

$$
\begin{gathered}
\widehat{X}_{t_{k+1}}^{N}\left(t_{k}, x\right)=x+\bar{u}^{N}\left(t_{k+1}, x\right) \cdot h+\sqrt{2 \nu} \cdot \sqrt{h} q\left(\frac{W_{t_{k+1}}-W_{t_{k}}}{\sqrt{h}}\right), \\
\widehat{Y}_{t_{k}}^{N}\left(t_{k}, x\right)=\mathbb{E}\left[\bar{u}^{N}\left(t_{k+1}, \widehat{\Pi}_{\delta}\left(\widehat{X}_{t_{k+1}}^{N}\left(t_{k}, x\right)\right)\right)\right], \\
\widehat{Q}^{N}\left(t_{k+1}, x\right)=\mathbb{E}\left[\sum_{\ell=1}^{N_{R}} \frac{3}{2\left(\sqrt{s_{\ell}}\right)^{3}} \bar{u}_{i}^{N} \cdot \bar{u}_{j}^{N}\left(t_{k+1}, \widehat{\Pi}_{\delta}\left(x+\sqrt{s_{\ell}}\left[q\left(\bar{\xi}_{s_{\ell}}\right)+q\left(\widetilde{\xi}_{s_{\ell}}\right)+q\left(\widehat{\xi}_{s_{\ell}}\right)\right]\right)\right)\right. \\
\left.\quad \times q^{i}\left(\bar{\xi}_{s_{\ell}}\right) q^{j}\left(\widetilde{\xi}_{s_{\ell}}\right) q\left(\widehat{\xi}_{s_{\ell}}\right) \cdot \tau\right], \\
\bar{u}^{N}\left(t_{k}, x\right)=\widehat{Y}_{t_{k}}^{N}\left(t_{k}, x\right)+\left[\widehat{Q}^{N}\left(t_{k+1}, x\right)-f\left(T-t_{k+1}, x\right)\right] \cdot h .
\end{gathered}
$$

Remark 2. The Algorithm 3.4 involves the approximation of the velocity field for each position. In order to reduce the computing time, the velocity field $\bar{u}^{N}\left(t_{k}, \cdot\right)$ can be constructed by using a parallel computer implementation based on a space partition. 


\section{Numerical tests}

Let $u(t, x)=\left(u_{1}(t, x), \ldots, u_{d}(t, x)\right)^{\top}$ be the exact velocity field that solves the incompressible Navier-Stokes equations (1). Since the equivalent backward form (8), by abuse of notation, we denote in the same way $\bar{u}^{N}(t, x)=\left(\bar{u}_{1}^{N}(t, x), \ldots, \bar{u}_{d}^{N}(t, x)\right)^{\top}$ the estimation of $u(t, x)$ obtained from Algorithm 3.4 i.e. by means of Algorithm 3.3 with $Q^{N}$ estimated as in (20). As in Algorithm 3.1. we only consider discretization-steps $\delta, h \in(0,1)$ such that $\delta<h$. The pressure term is computed with step sizes $\tau \in(0,1)$ to the truncated interval $\left[\frac{1}{3 N}, \frac{N}{3}\right]$, and we take spatial steps $\delta<\frac{1}{\sqrt{N}}$. The data of the $L^{2}$-optimal $Q$-quantizer of the $\mathcal{N}\left(0, I_{d}\right)$ distribution are provided by Corlay, Pagès and Printems [20. We consider the estimation errors

$$
e\left(t_{k}\right):=\sup _{x \in \mathcal{C}_{\delta}}\left\|u\left(t_{k}, x\right)-\bar{u}^{N}\left(t_{k}, x\right)\right\|^{2}
$$

for each $k \in\left\{1, \ldots, N_{T}\right\}$ and

$$
e_{i}\left(t_{k}\right):=\sup _{x \in \mathcal{C}_{\delta}}\left|u_{i}\left(t_{k}, x\right)-\bar{u}_{i}^{N}\left(t_{k}, x\right)\right|^{2} \quad ; \forall i \in\{1, \ldots, d\} .
$$

\subsection{Taylor-Green vortex}

We begin by studying the Taylor-Green vortices introduced by G. I. Taylor and A. E. Green in 1937 [66]. It is a classical turbulence model helpful to test numerical schemes for the incompressible Navier-Stokes equations. More precisely, we consider the vortex flow given by the incompressible Navier-Stokes equations (1) without external force field and initial condition

$$
\left\{\begin{array}{l}
g_{1}(x)=\frac{2}{\sqrt{3}} \sin \left(\theta+\frac{2 \pi}{3}\right) \sin \left(x_{1}\right) \cos \left(x_{2}\right) \cos \left(x_{3}\right), \\
g_{2}(x)=\frac{2}{\sqrt{3}} \sin \left(\theta-\frac{2 \pi}{3}\right) \cos \left(x_{1}\right) \sin \left(x_{2}\right) \cos \left(x_{3}\right), \\
g_{3}(x)=\frac{2}{\sqrt{3}} \sin (\theta) \cos \left(x_{1}\right) \cos \left(x_{2}\right) \sin \left(x_{3}\right)
\end{array}\right.
$$

where $x=\left(x_{1}, x_{2}, x_{3}\right)^{\top}$ and $\theta \in[0,2 \pi]$. In particular, by a shift of origin $(0,0,0) \rightarrow(\pi / 2, \pi / 2,0)$ and choosing $\theta=0$ and $x_{3} \equiv 0$, we have the explicit $2 d$ Taylor-Green vortex flow

$$
\left\{\begin{array}{l}
u_{1}(t, x)=-\cos \left(x_{1}\right) \sin \left(x_{2}\right) \exp (-2 \nu t), \\
u_{2}(t, x)=\sin \left(x_{1}\right) \cos \left(x_{2}\right) \exp (-2 \nu t), \\
p(t, x)=-\frac{1}{4}\left(\cos \left(2 x_{1}\right)+\cos \left(2 x_{2}\right)\right) \exp (-4 \nu t),
\end{array}\right.
$$

for $x \in[0,2 \pi]^{2}$, that solves the incompressible Navier-Stokes equations (1) with viscosity parameter $\nu>0$ and $f \equiv 0$. The velocity $u$ decreases its magnitude through time by the exponential factor $e^{-2 \nu t}$ for $t>0$ [7].

The exact solution (21) gives us a benchmark to Algorithm 3.4 with respect to a finite difference method [13, 14]. As in [13], we estimate numerically (21) with viscosity parameter $\nu=1$. We apply Algorithm 3.4 by using the truncation parameter $N=6$, time-step $h=1 / 40$, space-discretization step $\delta=\pi / 126$ and $Q=6$ quantization points, until a final time $T=10 h$. We compute the term $\widehat{Q}^{N}$ by taking a left Riemann sum approximation of the integrals using $N_{R}=\frac{\frac{N}{3}-\frac{1}{3 N}}{\tau}=18$ uniform subintervals of $\left[\frac{1}{3 N}, \frac{N}{3}\right]$. Table 1 depicts the numerical results. The discretization steps are related linearly, instead of the restrictive assumption $h=\mathcal{O}\left(\delta^{2}\right)$ in the case of the finite-difference scheme [14]. 


\begin{tabular}{|c|c|c|c|c|c|c|c|c|c|c|}
\hline \multicolumn{10}{|c|}{ Algorithm 3.4} & \multicolumn{3}{|c|}{} \\
\hline$k$ & 1 & 2 & 3 & 4 & 5 & 6 & 7 & 8 & 9 & 10 \\
\hline$\hat{e}_{1}\left(t_{k}\right)$ & 0.2080 & 0.6509 & 1.2064 & 1.8993 & 2.6558 & 3.3847 & 4.0197 & 4.6681 & 5.4006 & 6.0757 \\
\hline$\hat{e}_{2}\left(t_{k}\right)$ & 0.2129 & 0.6627 & 1.3068 & 2.0936 & 2.9979 & 3.9497 & 4.9062 & 5.8747 & 6.8223 & 7.7452 \\
\hline$\hat{e}\left(t_{k}\right)$ & 0.2139 & 0.6628 & 1.3153 & 2.1040 & 3.0136 & 3.9691 & 4.9306 & 5.9109 & 6.8528 & 7.7768 \\
\hline
\end{tabular}

Table 1: Estimation errors $\hat{e}\left(t_{k}\right):=e\left(t_{k}\right) \cdot 10^{3}$ and $\hat{e}_{i}\left(t_{k}\right):=e_{i}\left(t_{k}\right) \cdot 10^{3}$ to the two-dimensional Taylor-Green vortex performed by Algorithm 3.4 with $N=6, h=1 / 40, \delta=\pi / 126, Q=6$ and $N_{R}=18$.

\subsection{Beltrami flows}

The Beltrami flows, introduced by E. Beltrami in 1889 [3], are the ones for which the vorticity vector $\omega=\operatorname{curl} u$, with rotational operator curl $:=\nabla \times$, satisfies $\omega=\lambda u$ for some parameter $\lambda$. That is, the vorticity and the velocity vectors are aligned, or well the velocity is an eigenfunction of the rotational operator.

\subsubsection{Three-dimensional Beltrami flow}

In particular, we study the three-dimensional viscous Beltrami flow

$$
\left\{\begin{array}{l}
u(t, x)=g(x) e^{-\nu \lambda^{2} t} \\
p(t, x)=p_{s}-\rho\left[\frac{\|u(t, x)\|^{2}}{2}+\mathrm{g} x_{3}\right]
\end{array}\right.
$$

with non-divergence initial velocity field

$$
\left\{\begin{array}{l}
g_{1}(x)=-\frac{A}{k^{2}+\ell^{2}}\left[\lambda \ell \cos \left(k x_{1}\right) \sin \left(\ell x_{2}\right) \sin \left(m x_{3}\right)+m k \sin \left(k x_{1}\right) \cos \left(\ell x_{2}\right) \cos \left(m x_{3}\right)\right] \\
g_{2}(x)=\frac{A}{k^{2}+\ell^{2}}\left[\lambda k \sin \left(k x_{1}\right) \cos \left(\ell x_{2}\right) \sin \left(m x_{3}\right)-m \ell \cos \left(k x_{1}\right) \sin \left(\ell x_{2}\right) \cos \left(m x_{3}\right)\right] \\
g_{3}(x)=A \cos \left(k x_{1}\right) \cos \left(\ell x_{2}\right) \sin \left(m x_{3}\right),
\end{array}\right.
$$

where $x=\left(x_{1}, x_{2}, x_{3}\right)^{\top}, p_{s} \geq 0$ a time-independent stagnation pressure at ground level, $A$ the amplitude of the vertical velocity and Beltrami coefficient

$$
\lambda=\sqrt{k^{2}+\ell^{2}+m^{2}} .
$$

Here, $\mathrm{g}>0$ is the acceleration due to gravity and $\rho>0$ is the density of the fluid which is assumed, as the kinematic viscosity $\nu>0$, to be constant. The Beltrami flow (22) solves the incompressible Navier-Stokes equations (1) with $\nu>0$, external force field $f(t, x)=-\mathrm{g}(0,0,1)^{\top}$ and initial divergence-free vector field $g(x)$ as above (see [61]). Defining the potential $\phi(t, x)=$ - $\mathrm{g} x_{3}$, observe that $f(t, x)=\nabla \phi(t, x)$. Thus, the constant density $\rho>0$ and the conservative external force field $f$ are considered as part of the pressure term $p(t, x)$. That is

$$
\left\{\begin{array}{l}
u(t, x)=g(x) e^{-\nu \lambda^{2} t} \\
p(t, x)=\frac{p_{s}}{\rho}-\left[\frac{\|u(t, x)\|^{2}}{2}+\mathrm{g} x_{3}-\phi(t, x)\right]=\frac{p_{s}}{\rho}-\frac{\|u(t, x)\|^{2}}{2}
\end{array}\right.
$$

for $t \in[0, T]$ and $x \in\left[0, \frac{2 \pi}{k}\right] \times\left[0, \frac{2 \pi}{\ell}\right] \times\left[0, \frac{2 \pi}{m}\right]$, solves the incompressible Navier-Stokes equations (1) with viscosity parameter $\nu>0$ and external force $f \equiv 0$.

We test Algorithm 3.4 by solving the Beltrami flow (23) with different combinations of the parameters $\nu>0, A>0$ and $k=\ell=m=1$ until a final time $T>0$. For these simulations, the 
Reynolds number is in the range $[1,200]$. The parameters are described below. The time-step and the final time are such that the exponential damping factor cannot be neglected.

First, we perform one-step in time estimations by taking discretization steps $0<\delta=\frac{2 \pi}{N_{\delta}}<$ $h=\frac{T}{N_{h}}$ with $N_{h}=1$. As in the two dimensional context, we fix the truncation parameter $N=6$ and numerical quantities $Q=6$ and $N_{R}=18$. Table 2 depicts the estimation errors. Observe the influence of the viscosity $\nu>0$ and the velocity amplitude parameter $A>0$ to the quality of aproximations.

\begin{tabular}{|c|c|c|c|c|c|c|c|}
\hline$\nu$ & $A$ & $h$ & $N_{\delta}$ & $e_{1}(h)$ & $e_{2}(h)$ & $e_{3}(h)$ & $e(h)$ \\
\hline 0.005 & 0.5 & 0.1 & 63 & 0.0000807 & 0.0000707 & 0.0000994 & 0.000127 \\
\hline 0.005 & 1 & 0.1 & 63 & 0.00117 & 0.000777 & 0.00130 & 0.00136 \\
\hline 0.005 & 2 & 0.1 & 63 & 0.01234 & 0.01083 & 0.01867 & 0.01909 \\
\hline 0.005 & 5 & 0.1 & 63 & 0.6691 & 0.5030 & 0.94500 & 0.9840 \\
\hline 0.01 & 0.5 & 0.1 & 63 & 0.0001203 & 0.0001317 & 0.0001631 & 0.0001869 \\
\hline 0.01 & 1 & 0.1 & 63 & 0.001217 & 0.0008113 & 0.001019 & 0.001809 \\
\hline 0.01 & 2 & 0.1 & 63 & 0.01352 & 0.01183 & 0.01888 & 0.01912 \\
\hline 0.01 & 5 & 0.1 & 63 & 0.6216 & 0.4888 & 0.9545 & 0.9903 \\
\hline 0.1 & 0.5 & 0.2 & 40 & 0.0009937 & 0.0007624 & 0.0006606 & 0.0011590 \\
\hline 0.1 & 1 & 0.2 & 40 & 0.0046 & 0.0029 & 0.0046 & 0.0055 \\
\hline 0.1 & 2 & 0.2 & 40 & 0.0439 & 0.0265 & 0.0635 & 0.0654 \\
\hline 0.1 & 5 & 0.2 & 40 & 4.0336 & 2.4247 & 5.8554 & 5.8991 \\
\hline 0.1 & 0.5 & 0.1 & 63 & 0.0003391 & 0.0002831 & 0.0002316 & 0.0004004 \\
\hline 0.1 & 1 & 0.1 & 63 & 0.001482 & 0.001242 & 0.002362 & 0.002489 \\
\hline 0.1 & 2 & 0.1 & 63 & 0.016590 & 0.010939 & 0.019589 & 0.020716 \\
\hline 0.1 & 5 & 0.1 & 63 & 0.5767 & 0.4280 & 0.8913 & 0.9089 \\
\hline 1 & 0.5 & 0.2 & 40 & 0.0056 & 0.0035 & 0.0042 & 0.0062 \\
\hline 1 & 1 & 0.2 & 40 & 0.0180 & 0.0160 & 0.0203 & 0.0247 \\
\hline 1 & 2 & 0.2 & 40 & 0.0928 & 0.0575 & 0.0798 & 0.0988 \\
\hline 1 & 5 & 0.2 & 40 & 1.0389 & 1.0320 & 1.1466 & 1.6058 \\
\hline 1 & 0.5 & 0.1 & 63 & 0.0015 & 0.0023 & 0.0024 & 0.0028 \\
\hline 1 & 1 & 0.1 & 63 & 0.0075 & 0.0085 & 0.0118 & 0.0136 \\
\hline 1 & 2 & 0.1 & 63 & 0.0401 & 0.0423 & 0.0514 & 0.0612 \\
\hline 1 & 5 & 0.1 & 63 & 0.5353 & 0.3616 & 0.4363 & 0.6380 \\
\hline 1 & 1 & 0.05 & 126 & 0.0025 & 0.0027 & 0.0033 & 0.0037 \\
\hline 1 & 5 & 0.05 & 126 & 0.1557 & 0.1317 & 0.1640 & 0.1816 \\
\hline 1 & 1 & 0.04 & 158 & 0.0016 & 0.0018 & 0.0026 & 0.0027 \\
\hline 1 & 5 & 0.04 & 158 & 0.1177 & 0.0844 & 0.1293 & 0.1353 \\
\hline 1 & 1 & 0.025 & 252 & 0.00073 & 0.00069 & 0.00095 & 0.00098 \\
\hline 1 & 5 & 0.025 & 252 & 0.0476 & 0.0386 & 0.0599 & 0.0602 \\
\hline
\end{tabular}

Table 2: One-step in time estimation errors of the Algorithm $3.4\left(h>0\right.$ and $\left.\delta=\frac{2 \pi}{N_{\delta}}>0\right)$ for the numerical solution of the Beltrami flow $(23)(\nu>0, A>0, k=l=m=1)$.

Now, we consider the numerical approximation of the $3 d$ space-periodic velocity field $u$ of the Beltrami flow (23) with $\nu=0.1, A=0.5, k=\ell=m=1$ until the final time $T=2$. Note that the parameters $\rho, \mathrm{g}$ and $p_{s}$ have no influence on the results. In this case, the exponential factor reduces the magnitude of the initial velocity field until about $54.88 \%$. Table 3 shows the performance of Algorithm 3.4 with truncation parameter $N=6$, time-step $h=T / 10$, spatial discretization $\delta=\pi / 20, Q=6$ quantization points and $N_{R}=18$ subintervals to the Riemann sum estimations.

Finally, we numerically solve of the $3 d$ space-periodic velocity field $u$ of the Beltrami flow (23) with $\nu=0.1, A=1, k=\ell=m=1$ until $T=1$. Observe that the exponential factor reduces the magnitude of the initial velocity field until about $74.08 \%$. Table 4 shows the performance of Algorithm 3.4 with $N=6, h=T / 10, \delta=2 \pi / 63, Q=6$ and $N_{R}=18$. 


\begin{tabular}{|c|c|c|c|c|c|c|c|c|c|c|}
\hline \multicolumn{9}{|c|}{ Algorithm 3.4} & \multicolumn{3}{|c|}{} \\
\hline$k$ & 1 & 2 & 3 & 4 & 5 & 6 & 7 & 8 & 9 & 10 \\
\hline$\tilde{e}_{1}\left(t_{k}\right)$ & 0.0994 & 0.2719 & 0.5046 & 0.7557 & 1.0427 & 1.3278 & 1.6278 & 1.8934 & 2.1408 & 2.3464 \\
\hline$\tilde{e}_{2}\left(t_{k}\right)$ & 0.0762 & 0.2097 & 0.3906 & 0.5483 & 0.7490 & 0.8825 & 1.0559 & 1.2134 & 1.3037 & 1.3780 \\
\hline$\tilde{e}_{3}\left(t_{k}\right)$ & 0.0661 & 0.1944 & 0.3166 & 0.4872 & 0.6616 & 0.7902 & 1.0241 & 1.1166 & 1.2321 & 1.3769 \\
\hline$\tilde{e}\left(t_{k}\right)$ & 0.1159 & 0.2954 & 0.5503 & 0.8002 & 1.1048 & 1.4035 & 1.6854 & 1.9386 & 2.1779 & 2.4098 \\
\hline
\end{tabular}

Table 3: Estimation errors $\tilde{e}\left(t_{k}\right):=e\left(t_{k}\right) \cdot 10^{2}$ and $\tilde{e}_{i}\left(t_{k}\right):=e_{i}\left(t_{k}\right) \cdot 10^{2}$ to the three-dimensional Beltrami flow $(23)(\nu=0.1, A=0.5, k=l=m=1)$ by using Algorithm $3.4(N=6, h=1 / 5$, $\delta=\pi / 20, Q=6$ and $\left.N_{R}=18\right)$.

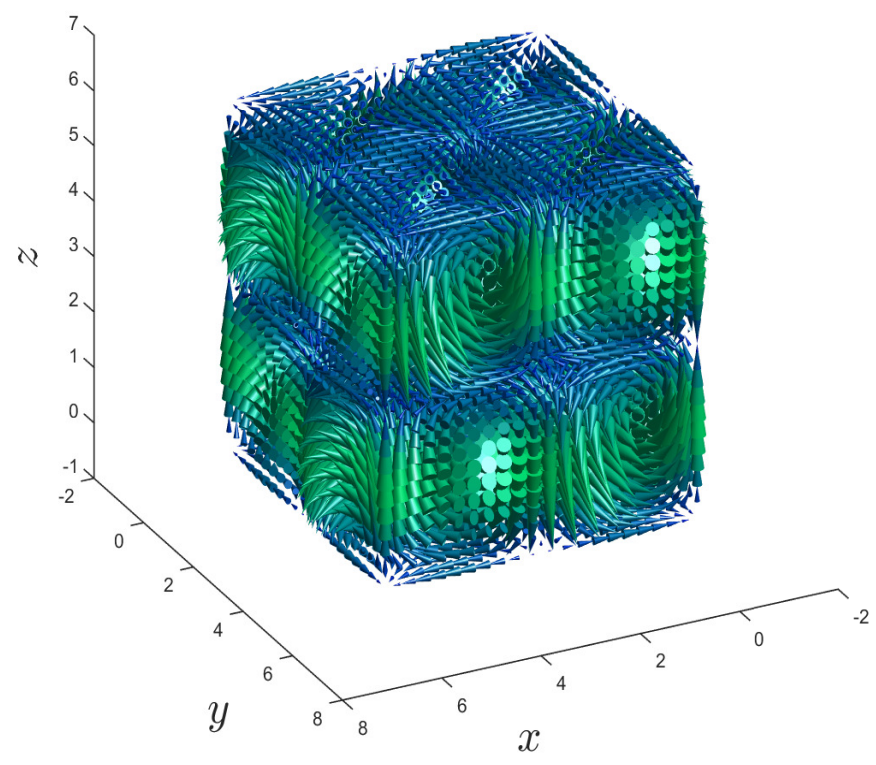

Figure 1: Initial velocity field to the three-dimensional Beltrami flow (22) with $\nu=0.1, A=1$ and $k=\ell=m=1$.

\begin{tabular}{|c|c|c|c|c|c|c|c|c|c|c|}
\hline \multicolumn{10}{|c|}{ Algorithm 3.4} \\
\hline$k$ & 1 & 2 & 3 & 4 & 5 & 6 & 7 & 8 & 9 & 10 \\
\hline$\tilde{e}_{1}\left(t_{k}\right)$ & 0.1482 & 0.4451 & 0.8280 & 1.3023 & 1.7570 & 2.3748 & 2.9724 & 3.6903 & 4.3578 & 5.0886 \\
\hline$\tilde{e}_{2}\left(t_{k}\right)$ & 0.1242 & 0.3675 & 0.6741 & 1.0009 & 1.4126 & 1.8552 & 2.3155 & 2.9924 & 3.5432 & 4.1730 \\
\hline$\tilde{e}_{3}\left(t_{k}\right)$ & 0.2362 & 0.7134 & 1.3252 & 2.0755 & 2.9842 & 3.9512 & 4.9251 & 5.9950 & 7.0374 & 7.9639 \\
\hline$\tilde{e}\left(t_{k}\right)$ & 0.2489 & 0.7825 & 1.4721 & 2.3394 & 3.2192 & 4.2533 & 5.2970 & 6.3564 & 7.3176 & 8.2782 \\
\hline
\end{tabular}

Table 4: Estimation errors $\tilde{e}\left(t_{k}\right):=e\left(t_{k}\right) \cdot 10^{2}$ and $\tilde{e}_{i}\left(t_{k}\right):=e_{i}\left(t_{k}\right) \cdot 10^{2}$ to the three-dimensional Beltrami flow (23) $(\nu=0.1, A=1, k=l=m=1)$ by using Algorithm $3.4(N=6, h=1 / 10$, $\delta=2 \pi / 63, Q=6$ and $\left.N_{R}=18\right)$. 

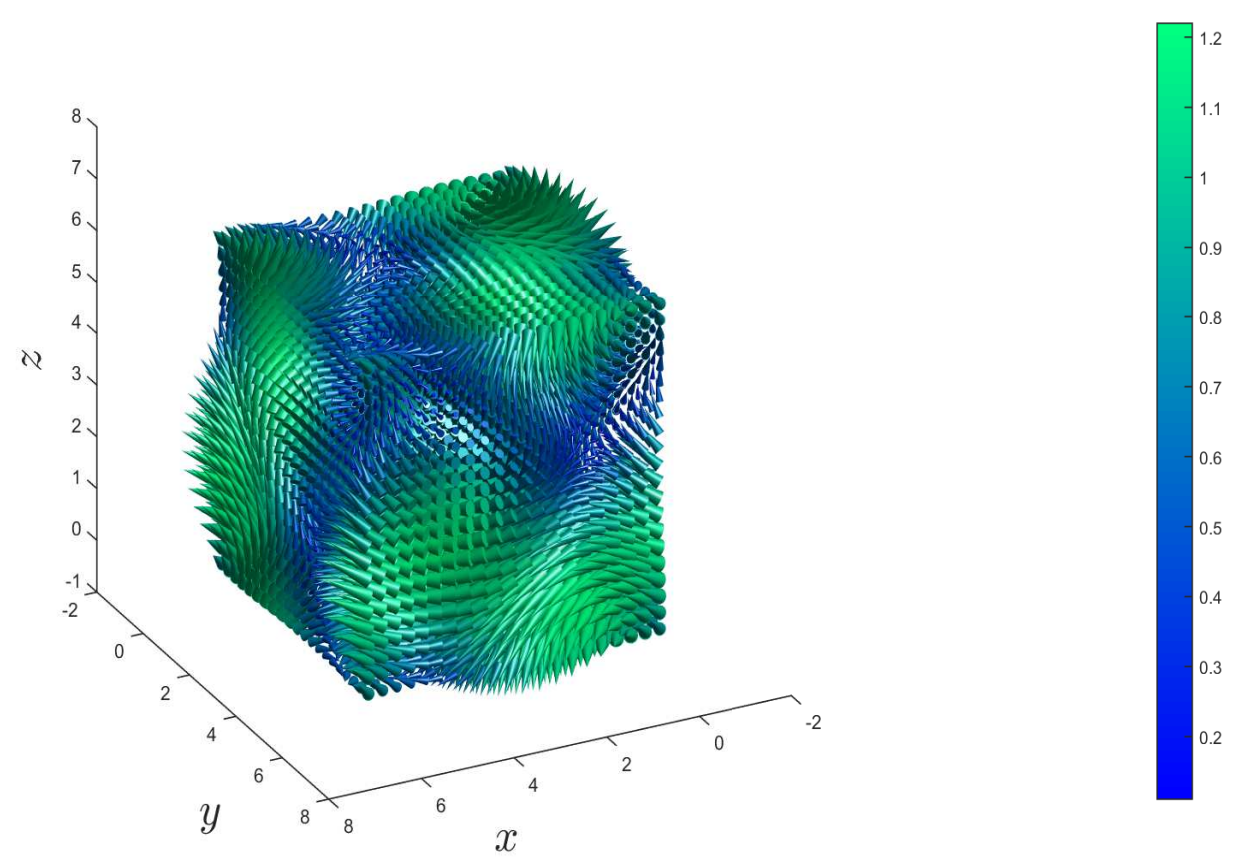

Figure 2: Initial velocity field to the three-dimensional ABC flow (24) with $\nu=0.01$ and $A=B=C=0.5$.

\subsubsection{Arnold-Beltrami-Childress flow}

Now, we consider the Arnold-Beltrami-Childress flow [44]

$$
\left\{\begin{array}{l}
u_{1}(t, x)=\left(A \sin \left(x_{3}\right)+C \cos \left(x_{2}\right)\right) \exp (-\nu t) \\
u_{2}(t, x)=\left(B \sin \left(x_{1}\right)+A \cos \left(x_{3}\right)\right) \exp (-\nu t) \\
u_{3}(t, x)=\left(C \sin \left(x_{2}\right)+B \cos \left(x_{1}\right)\right) \exp (-\nu t) \\
p(t, x)=-\left(B C \cos \left(x_{1}\right) \sin \left(x_{2}\right)+A B \sin \left(x_{1}\right) \cos \left(x_{3}\right)+A C \sin \left(x_{3}\right) \cos \left(x_{2}\right)\right) \exp (-2 \nu t)+c
\end{array}\right.
$$

for $(t, x) \in[0, T] \times[0,2 \pi]^{3}$, with final time $T>0$, parameters $A, B, C \in \mathbb{R}$ and constant $c \in \mathbb{R}$. The ABC flow was introduced by Arnold [2] and Childress [11] as a class of Beltrami flow (with Beltrami coefficient $\lambda=1$ ). The three-dimensional velocity field $u$ and pressure term $p$ defined by (24) solve the incompressible Navier-Stokes equations (1) with viscosity parameter $\nu>0$ and external force $f \equiv 0$. In our simulations, we consider a Reynolds number in the range $[1,100]$.

We examine the ABC flow (24) with $\nu=1$ and $A=B=C=1$ until a final time $T>0$. The Algorithm 3.4 is performed to obtain one-step in time estimations (that is $T=h)$ by using the time steps $h=1 / 10,1 / 20,1 / 30,1 / 40$ and the space discretizations $\delta=2 \pi / 63,2 \pi / 126,2 \pi / 189,2 \pi / 252$, respectively. The estimation errors result

$$
e(h)=4.3185 \cdot 10^{-2}, 1.1998 \cdot 10^{-2}, 5.2584 \cdot 10^{-3}, 3.3223 \cdot 10^{-3},
$$

respectively. As above we have set the parameters $N=6, Q=6$ and $N_{R}=18$. Observe the better results as the time-space grid increases its quantity of nodes. The order of error estimations results similar as in the case of the discontinuous Galerkin method presented in 65 .

Finally, we simulate the ABC flow (24) with $\nu=0.01$ and $A=B=C=0.5$ until $T=7 / 10$. Table 5 presents the estimation errors of Algorithm 3.4 with truncation parameter 


\begin{tabular}{|c|c|c|c|c|c|c|c|c|c|c|}
\hline \multicolumn{10}{|c|}{ Algorithm 3.4} & \multicolumn{1}{|c|}{} \\
\hline$k$ & 1 & 2 & 3 & 4 & 5 & 6 & 7 & 8 & 9 & 10 \\
\hline$\tilde{e}_{1}\left(t_{k}\right)$ & 0.0400 & 0.1449 & 0.3178 & 0.5553 & 0.8433 & 1.1800 & 1.5744 & 2.0311 & 2.5400 & 3.0662 \\
\hline$\tilde{e}_{2}\left(t_{k}\right)$ & 0.0574 & 0.1990 & 0.4256 & 0.7479 & 1.1740 & 1.6526 & 2.3021 & 2.9373 & 3.8502 & 4.6322 \\
\hline$\tilde{e}_{3}\left(t_{k}\right)$ & 0.0427 & 0.1564 & 0.3434 & 0.5954 & 0.9202 & 1.3296 & 1.8393 & 2.3489 & 3.0317 & 3.7326 \\
\hline$\tilde{e}\left(t_{k}\right)$ & 0.1001 & 0.3661 & 0.8010 & 1.3877 & 2.1203 & 3.0003 & 4.1640 & 5.2858 & 6.9379 & 8.6758 \\
\hline
\end{tabular}

Table 5: Estimation errors $\tilde{e}\left(t_{k}\right):=e\left(t_{k}\right) \cdot 10^{2}$ and $\tilde{e}_{i}\left(t_{k}\right):=e_{i}\left(t_{k}\right) \cdot 10^{2}$ to the three-dimensional ABC flow (24) $(\nu=0.01, A=B=C=0.5)$ by using Algorithm $3.4(N=6, h=7 / 100$, $\delta=\pi / 45, Q=6$ and $\left.N_{R}=18\right)$.

$N=6$, time-step $h=T / 10$, spatial discretization $\delta=\pi / 45, Q=6$ quantization points and $N_{R}=18$ subintervals to the Riemann sum estimations.

\section{Conclusions}

Forward-backward SDEs driven by Brownian motion are linked to nonlinear PDEs by means of the Feynman-Kac formula. The deterministic solution is interpreted as the conditional expectation of a diffusion process, and a stochastic algorithm to compute estimations is deduced from its probabilistic representation. A novel system of FBSDEs due to F. Delbaen, J. Qiu and S. Tang [26] introduces a probabilistic approach associated to the incompressible NavierStokes equations in dimensions $d=2,3$. The Burgers equation is bounded to Itô and Backward SDEs on $[0, T]$. Then the pressure term and the incompressibility condition are incorporated by an additional BSDE on the time interval $(0,+\infty)$. Therefore an estimation of the velocity field that solves the unsteady Navier-Stokes equations follows from the numerical simulation of stochastic particles governed by a coupled forward-backward SDEs system (FBSDS) driven by independent Brownian motions.

Motivated by Delbaen et al. the infinite interval of the extra BSDE is truncated to $\left[\frac{1}{N}, N\right]$; with $N \in \mathbb{N}$, thus the velocity field is approximated by $u^{N}$. The resulting FBSDEs system is computationally simulated by positioning particles onto an uniform temporal-spatial grid with discretization parameters in time $h>0$ and space $\delta>0$ such that $h, \delta \in(0,1)$. The forward-backward SDEs are numerically solved by using a methodology in the spirit of Delarue and Menozzi [24 i.e. by means a local discretization in time and taking Euler type integration schemes and optimal quantizers to the Wiener increments to approximate the conditional expectations on each temporal-spatial node. The pressure term demands to solve the BSDE associated to its Poisson problem, and the approximation of expectations of integrals involving path of Brownian motions appears as an additional challenge. As in [24], the spatial steps are considered $\delta<h$. Moreover we have taken $\delta<\frac{1}{\sqrt{N}}$ to capture the local perturbations for solving the extra BSDE. In our numerical tests, the quantization of the underlying Gaussian processes together with a Riemann sum estimation of integrals appear as a good approach to deal with this problem. The integrals on the truncated interval $\left[\frac{1}{3 N}, \frac{N}{3}\right]$ are approximated by taking Riemann sum approximations with step sizes $\tau \in(0,1)$.

In this work, we study the numerical simulation of spatially periodic solutions to the NavierStokes equations. We present a two dimensional Taylor-Green vortex simulation with order $10^{-3}$ estimation errors to the velocity field. Moreover we report numerical results on three dimensional Beltrami flows achieving order $10^{-2}$ for the approximation errors. The quantity of particles increases as the space dimension grows, and additional computation is demanded to simulate the exact solutions. Moreover, from Morrey's inequality

$$
H^{m} \subset C^{m-\left[\frac{d}{2}\right]-1, \alpha},
$$


with Hölder exponent $\alpha=\frac{1}{2}$ when $d=3$ or any number $\alpha \in(0,1)$ if $d=2$. Observe a loss of accuracy to the order of estimation $\mathcal{O}\left(1 / N^{\frac{\alpha}{4}}\right)$ in the three dimensional context, hence the truncation parameter $N$ must increases to obtain theoretical errors as in the two dimension case.

The expected values are computed by replacing the underlying Gaussian random variables with optimal quantizers of the Normal distribution available in the website [20]. In practice, the quantization approach presents an advantage to the traditional Monte Carlo simulation because its non-asymptotic error bound of estimation $\mathcal{O}\left(1 / Q^{\frac{1}{d}}\right)$. Given a codebook of size $Q$, the quantization error is better in low dimension. We consider a few quantizer points instead of the usage of many independent realizations of random variables.

We report numerical results by using the truncation parameter $N=6$ and $Q=6$ optimal quantizer points in two and three dimensions. The Riemann sum estimations on the interval $\left[\frac{1}{3 N}, \frac{N}{3}\right]$ are computed by using the uniform discretization step $\tau=\left(\frac{N}{3}-\frac{1}{3 N}\right) / N_{R}$ with $N_{R}=$ 18 subintervals. The Euler-Maruyama method is considered to approximate the Itô SDEs components with additive noise $\nu \in\{1,0.1,0.01,0.005\}$. The time discretization step is essential to the performance of numerical solutions. It is well-known that Euler-Maruyama scheme could not be appropriate for turbulent flows as in the case of passive tracers model with low viscosity parameter. A convenient numerical scheme needs to be considered in the case of vanishing viscosity (see e.g. [60]).

The rate of convergence of Algorithm 3.4 depends on the initial data $f, g$ and $\nu>0$, the parameter $N$, the discretization steps on $[0, T], \mathbb{R}^{d}$ and $\left[\frac{1}{3 N}, \frac{N}{3}\right]$, and the optimal quantization of the independent Brownian motions $W$ and $B$. Roughly we have

$$
\left\|u-\bar{u}^{N}\right\| \leq\left\|u-u^{N}\right\|+\left\|u^{N}-\bar{u}^{N}\right\|=\mathcal{O}\left(\frac{1}{N^{\frac{\alpha}{4}}}\right)+\operatorname{error}(N, h, \delta, \tau, Q),
$$

with $\alpha$ depending on $d \in\{2,3\}$. The estimation error involves the dimension $d$ and the $\alpha$-Hölder regularity. The reported numerical tests motivate the theoretical study and numerical analysis of the probabilistic methodology associated to the incompressible Navier-Stokes equations. Moreover they suggest the extension of variance reduction methods to contexts as computing conditional expectations to solve systems of FBSDEs. Dealing with more general boundary conditions will be subject to future works.

\section{Acknowledgements}

H. Mardones González thanks the funding of the Becas Chile, CONICYT grant, Chile, and the partial support by research team TOSCA, INRIA Nancy Grand Est, France, and ANESTOC TOSCA associated team INRIA project.

\section{References}

[1] F. Antonelli. Backward-forward stochastic differential equations. Ann. Appl. Probab., 3:777-793, 1993.

[2] V. I. Arnold. Sur la topologie des écoulements stationnaires des fluides parfaits. C. $R$. Acad. Sc. Paris, 261:17-20, 1965.

[3] Eugenio Beltrami. Considerations on Hydrodynamics. Int. J. Fusion Energy, 3:53-57, 1985. Translated by Dr. Giuseppe Filipponi from original paper appeared in 1889 in Rendiconti del Reale Instítuto Lombardo, Series II, Vol. 22. 
[4] S. Benachour, B. Roynette, and P. Vallois. Branching process associated with 2d-Navier Stokes equation. Rev. Mat. Iberoam., 17:331-373, 2001.

[5] R. N. Bhattacharya, L. Chen, S. Dobson, R. B. Guenther, C. Orum, M. Ossiander, E. Thomann, and E. C. Waymire. Majorizing kernels and stochastic cascades with applications to incompressible Navier-Stokes equations. Trans. Amer. Math. Soc., 355:5003-5040, 2003.

[6] B. Bouchard and S. Menozzi. Strong approximations of BSDEs in a domain. Bernoulli, 15:1117-1147, 2009.

[7] M. E. Brachet, U. Frisch, D. I. Meiron, R. H. Morf, B. G. Nickel, and S. A. Orszag. Small-scale structure of the Taylor-Green vortex. J. Fluid Mech., 130:411-452, 1983.

[8] J. M. Burgers. A mathematical model illustrating the theory of turbulence. Adv. Appl. Mech., 1:171-199, 1948.

[9] J.-Y. Chemin, B. Desjardins, I. Gallagher, and E. Grenier. Mathematical Geophysics - An introduction to rotating fluids and the Navier-Stokes equations. Clarendon Press, Oxford, 2006.

[10] P. Cheridito, H. M. Soner, N. Touzi, and N. Victoir. Second-order backward stochastic differential equations and fully nonlinear parabolic PDEs. Comm. Pure Appl. Math., 60:1081-1110, 2007.

[11] S. Childress. New solutions of the kynematic dynamo problem. J. Math. Phys., 11:30633076, 1970.

[12] A. J. Chorin. A numerical method for solving incompressible viscous flow problems. $J$. Comput. Phys., 2:12-26, 1967.

[13] A. J. Chorin. Numerical solution of the Navier-Stokes equations. Math. Comp., 22:745-762, 1968.

[14] A. J. Chorin. On the convergence of discrete approximations to the Navier-Stokes equations. Math. Comp., 23:341-353, 1969.

[15] A. J. Chorin. Numerical study of slightly viscous flow. J. Fluid Mech., 57:785-796, 1973.

[16] A. J. Chorin. Vorticity and turbulence. Springer-Verlag, New York, corrected second printing edition, 1998.

[17] P. Constantin and G. Iyer. A stochastic Lagrangian representation of the three-dimensional incompressible Navier-Stokes equations. Comm. Pure Appl. Math., 61:330-345, 2008.

[18] P. Constantin and G. Iyer. A stochastic-Lagrangian approach to the Navier-Stokes equations in domains with boundary. Ann. Appl. Probab., 21:1466-1492, 2011.

[19] S. Corlay and G. Pagès. Functional quantization-based stratified sampling methods. Monte Carlo Methods Appl., 21:1-32, 2015.

[20] Sylvain Corlay, Gilles Pagès, and Jacques Printems. The optimal quantization website, 2005. http://www.quantize.maths-fi.com.

[21] M. G. Crandall, H. Ishii, and P.-L. Lions. User's guide to viscosity solutions of second order partial differential equations. Bull. Amer. Math. Soc., 27:1-67, 1992. 
[22] A. B. Cruzeiro and E. Shamarova. Navier-Stokes equations and forward-backward SDEs on the group of diffeomorphisms of a torus. Stoch. Proc. Appl., 119:4034-4060, 2009.

[23] F. Delarue. On the existence and uniqueness of solutions to FBSDEs in a non-degenerate case. Stoch. Proc. Appl., 99:209-286, 2002.

[24] F. Delarue and S. Menozzi. A forward-backward stochastic algorithm for quasi-linear PDEs. Ann. Appl. Probab., 16:140-184, 2006.

[25] F. Delarue and S. Menozzi. An interpolated stochastic algorithm for quasi-linear PDEs. Math. Comp., 77:125-158, 2008.

[26] F. Delbaen, J. Qiu, and S. Tang. Forward-backward stochastic differential systems associated to Navier-Stokes equations in the whole space. Stoch. Proc. Appl., 125:2516-2561, 2015 .

[27] Euler. Principes généraux du mouvement des fluides. Mém. Acad. Roy. Sci. Berlin, 11:274$315,1757$.

[28] C. L. Fefferman. Existence and smoothness of the Navier-Stokes equation. http://www. claymath.org/, 2000.

[29] R. P. Feynman. Space-time approach to non-relativistic quantum mechanics. Rev. Mod. Phys., 20:367-387, 1948.

[30] George S. Fishman. A first course in Monte Carlo. Duxbury Press, 2005.

[31] C. Foias, O. Manley, R. Rosa, and R. Teman. Navier-Stokes equations and turbulence, volume 83 of Encyclopedia of Mathematics and its Applications. Cambridge University Press, 2001.

[32] J. Fontbona. Stochastic vortex method for forced three-dimensional Navier-Stokes equations and pathwise convergence rate. Ann. Appl. Probab., 20:1761-1800, 2010.

[33] J. Fontbona and S. Méléard. A random space-time birth particle method for $2 d$ vortex equations with external fields. Math. Comp., 77(263):1525-1558, 2008.

[34] Siegfried Graf and Harald Luschgy. Foundations of quantization for probability distributions, volume 1730 of Lecture Notes in Mathematics. Springer-Verlag, Berlin, 2000.

[35] K. Itô. Stochastic integral. Proc. Imp. Acad. Tokyo, 20:519-524, 1944.

[36] K. Itô. On a stochastic integral equation. Proc. Japan Acad., 22:32-35, 1946.

[37] G. Iyer and J. Mattingly. A stochastic-Lagrangian particle system for the Navier-stokes equations. Nonlinearity, 21:2537-2553, 2008.

[38] M. Kac. On distributions of certain Wiener functionals. Trans. Amer. Math. Soc., 65(1):1$13,1949$.

[39] A. W. Kolkiewicz. Efficient Monte Carlo simulation for integral functionals of Brownian motion. J. Complexity, 30:255-278, 2014.

[40] Y. Le Jan and A. S. Sznitman. Stochastic cascades and 3-dimensional Navier-Stokes equations. Probab. Theory Relat. Fields, 109:343-366, 1997. 
[41] A. Lejay and V. Reutenauer. A variance reduction technique using a quantized Brownian motion as a control variate. J. Comput. Finance, 16:61-84, 2012.

[42] J. Ma and J. Yong. Forward-backward stochastic differential equations and their applications, volume 1702 of Lecture Notes in Mathematics. Springer-Verlag, Berlin-Heidelberg, 1999.

[43] Jin Ma, Philip Protter, and Jiong Min Yong. Solving forward-backward stochastic differential equations explicitly - a four step scheme. Probab. Theory Relat. Fields, 98(3):339-359, 1994.

[44] A. J. Majda and A. L. Bertozzi. Vorticity and incompressible flow. Cambridge University Press, Cambridge, 2002.

[45] X. Mao. Backward stochastic differential equations and quasilinear partial differential equations. In A. Etheridge, editor, Stochastic Partial Differential Equations, volume 216 of London Mathematical Society Lecture Note Series, pages 189-208, Cambridge, 1995. Cambridge University Press.

[46] H. A. Mardones González. Numerical solution of stochastic differential equations with multiplicative noise. PhD thesis, Universidad de Concepción, 2017. http://repositorio. udec.cl/handle/11594/2702.

[47] S. Méléard. Stochastic particle approximations for two-dimensional Navier-Stokes equations. In A. Maass, S. Martínez, and J. San Martín, editors, Dynamics and Randomness II, volume 10 of Nonlinear Phenomena and Complex Systems, pages 147-197. Kluwer Acad. Publ., Dordrecht, 2004.

[48] R. Mikulevicius and E. Platen. Rate of convergence of the Euler approximation for diffusion processes. Math. Nachr., 151:233-239, 1991.

[49] G. N. Milstein and M. V. Tretyakov. Discretization of forward-backward stochastic differential equations and related quasi-linear parabolic equations. IMA J. Numer. Anal., $27(1): 24-44,2007$.

[50] G. N. Milstein and M. V. Tretyakov. Solving the Dirichlet problem for Navier-Stokes equations by probabilistic approach. BIT Numer. Math., 52:141-153, 2012.

[51] G. N. Milstein and M. V. Tretyakov. Probabilistic methods for the incompressible NavierStokes equations with space periodic conditions. Adv. Appl. Prob., 45:742-772, 2013.

[52] C. Navier. Mémoire sur les lois du mouvement des fluides. Mem. Acad. Sci. Inst. France, 6:389-440, 1822.

[53] M. Ossiander. A probabilistic representation of solutions of the incompressible NavierStokes equations in $\mathbb{R}^{3}$. Probab. Theory Relat. Fields, 133:267-298, 2005.

[54] G. Pagès and J. Printems. Optimal quadratic quantization for numerics: the Gaussian case. Monte Carlo Methods Appl., 9:135-165, 2003.

[55] G. Pagès and J. Printems. Functional quantization for numerics with an application to option pricing. Monte Carlo Methods Appl., 11:407-446, 2005. 
[56] É. Pardoux. Backward stochastic differential equations and viscosity solutions of systems of semilinear parabolic and elliptic PDEs of second order. In L. Decreusefond, Jon Gjerde, B. Øksendal, and A. S. Üstünel, editors, Stochastic Analysis and Related Topics VI, volume 42 of Progress in Probability, pages 79-127, Boston, 1998. Birkhäuser.

[57] É. Pardoux and S. Peng. Backward stochastic differential equations and quasilinear parabolic partial differential equations. In B. L. Rozovskii and R. B. Sowers, editors, Stochastic Partial Differential Equations and Their Applications, volume 176 of Lect. Notes Control Inf. Sci., pages 200-217, Berlin-Heidelberg-New York, 1992. Springer-Verlag.

[58] É. Pardoux and S. G. Peng. Adapted solution of a backward stochastic differential equation. Syst. Control Lett., 14:55-61, 1990.

[59] É. Pardoux and S. Tang. Forward-backward stochastic differential equations and quasilinear parabolic PDEs. Probab. Theory Relat. Fields, 114:123-150, 1999.

[60] G. A. Pavliotis, A. M. Stuart, and K. C. Zygalakis. Calculating effective diffusivities in the limit of vanishing molecular diffusion. J. Comput. Phys., 228:1030-1055, 2009.

[61] A. Shapiro. The use of an exact solution of the Navier-Stokes equations in a validation test of a three-dimensional nonhydrostatic numerical model. Mon. Weather Rev., 121:24202425, 1993.

[62] G. G. Stokes. On the theories of the internal friction of fluids in motion, and of the equilibrium and motion of elastic solids. Trans. Camb. Phil. Soc., 8:287-319, 1849.

[63] D. Talay and O. Vaillant. A stochastic particle method with random weights for the computation of statistical solutions of McKean-Vlasov equations. Ann. Appl. Probab., 13(1):140-180, 2003.

[64] L. Tartar. An introduction to Navier-Stokes equation and Oceanography. Springer-Verlag, Berlin, Heidelberg, 2006.

[65] M. Tavelli and M. Dumbser. A staggered space-time discontinuous Galerkin method for the three-dimensional incompressible Navier-Stokes equations on unstructured tetrahedral meshes. J. Comput. Phys., 319:294-323, 2016.

[66] G. I. Taylor and A. E. Green. Mechanism of the production of small eddies from large ones. Proc. R. Soc. Lond. A, 158:499-521, 1937.

[67] E. C. Waymire. Probability \& incompressible Navier-Stokes equations: An overview of some recent developments. Probab. Surv., 2:1-32, 2005.

[68] X. Zhang. A stochastic representation for backward incompressible Navier-Stokes equations. Probab. Theory Relat. Fields, 148:305-332, 2010. 